JOURNAL OF THE AMERICAN MATHEMATICAL SOCIETY

Volume 13, Number 4, Pages 909-943

S 0894-0347(00)00341-6

Article electronically published on June 23, 2000

\title{
LINEAR ALGEBRAIC GROUPS AND COUNTABLE BOREL EQUIVALENCE RELATIONS
}

\author{
SCOT ADAMS AND ALEXANDER S. KECHRIS
}

This paper is a contribution to the study of Borel equivalence relations on standard Borel spaces (i.e., Polish spaces equipped with their Borel structure). In mathematics one often deals with problems of classification of objects up to some notion of equivalence by invariants. Frequently these objects can be viewed as elements of a standard Borel space $X$ and the equivalence turns out to be a Borel equivalence relation $E$ on $X$. A complete classification of $X$ up to $E$ consists of finding a set of invariants $I$ and a map $c: X \rightarrow I$ such that $x E y \Leftrightarrow c(x)=c(y)$. For this to be of any interest both $I$ and $c$ must be explicit or definable and as simple and concrete as possible. The theory of Borel equivalence relations studies the set-theoretic nature of possible invariants and develops a mathematical framework for measuring the complexity of such classification problems.

In organizing this study, the following concept of reducibility is fundamental. Let $E, F$ be equivalence relations on standard Borel spaces $X, Y$, resp. We say that $E$ is Borel reducible to $F$, in symbols,

$$
E \leq_{B} F,
$$

if there is a Borel map $f: X \rightarrow Y$ such that

$$
x E y \Leftrightarrow f(x) F f(y) .
$$

This simply means that any complete invariants for $F$ work as well for $E$ (after composing with $f$ ) and therefore, in some sense, $E$ has a classification problem at most as complicated as that of $F$. We also let

$$
E \sim_{B} F \Leftrightarrow E \leq_{B} F \& F \leq_{B} E,
$$

and this means that $E, F$ have classification problems of equal complexity. Finally, we use

$$
E<_{B} F \Leftrightarrow E \leq_{B} F \& F \not_{B} E
$$

to signify that $E$ has a (strictly) simpler classification problem than that of $F$.

Another way to look at this notion is to notice that $E \leq_{B} F$ simply means that there is an injection from the quotient space $X / E$ into $Y / F$ which is "Borel", in the sense that it has a Borel lifting. Thus $E \leq_{B} F$ can be interpreted as saying that $X / E$ has "Borel cardinality" less than or equal to that of $Y / F$ and similarly

Received by the editors March 27, 1999 and, in revised form, April 21, 2000.

2000 Mathematics Subject Classification. Primary 03E15; Secondary 37A20.

Key words and phrases. Borel equivalence relations, Borel reducibility, cocycles, superrigidity.

The first author's research was partially supported by NSF Grant DMS 9703480.

The second author's research was partially supported by NSF Grant DMS 9619880 and a Visiting Miller Research Professorship at U.C. Berkeley. 
$E \sim_{B} F$ means that $X / E, Y / F$ have the same "Borel cardinality", while $E<_{B} F$ means that $X / E$ has strictly smaller "Borel cardinality" than that of $Y / F$. Thus the study of the (pre-)order $\leq_{B}$ on Borel equivalence relations can also be viewed as the study of the "Borel cardinalities" of their quotient spaces.

In the beginning the structure of $\leq_{B}$ is quite simple. Denoting by $X$ also the equality relation on a standard Borel space $X$, and letting $n$ be any such space of finite cardinality $n$, we have that the following is an initial segment of $\leq_{B}$ :

$$
1<_{B} 2<_{B} 3<_{B} \cdots<_{B} \mathbb{N},
$$

and $\mathbb{N}<_{B} E$ for any Borel equivalence $E$ not in this sequence. The first nontrivial theorem concerning the structure of Borel equivalence relations under $\leq_{B}$ is the Silver Dichotomy (see Silver 32) which asserts that any Borel (in fact even coanalytic) equivalence relation on a Polish space has either countably many or else perfectly many equivalence classes, in the sense that there is a perfect set any two distinct elements of which belong to different equivalence classes. This shows that a strong form of the Continuum Hypothesis is valid for quotient spaces of Borel equivalence relations. It implies that

$$
1<_{B} 2<_{B} 3<_{B} \cdots<_{B} \mathbb{N}<_{B} \mathbb{R}
$$

is an initial segment of $\leq_{B}$ and $\mathbb{R}<_{B} E$ for any Borel equivalence relation $E$ not in this list.

For the next step, notice that the equivalence relations $E \leq_{B} \mathbb{R}$ are exactly those for which there is a Borel map $f: X \rightarrow Y$, where $X$ is the space of $E$ and $Y$ some standard Borel space, such that $x E y \Leftrightarrow f(x)=f(y)$. Such equivalence relations are called tame (or smooth or concretely classifiable as they can be completely classified by invariants which are members of some standard Borel space). The canonical example of a non-tame equivalence relation is the Vitali equivalence relation $E_{0}$ on $\mathbb{R}:$

$$
x E_{0} y \Leftrightarrow x-y \in \mathbb{Q} .
$$

Thus $\mathbb{R}<_{B} E_{0}$. The second dichotomy theorem for Borel equivalence relations, called the General Glimm-Effros Dichotomy (see Harrington-Kechris-Louveau [14]), asserts that for any Borel equivalence relation $E$, either $E$ is tame or else contains a (Borel) copy of $E_{0}$, and so $E_{0} \leq_{B} E$. (This generalizes earlier results of Glimm 12 and Effros [6].) Thus we have that

$$
1<_{B} 2<_{B} 3<_{B} \cdots<_{B} \mathbb{N}<_{B} \mathbb{R}<_{B} E_{0}
$$

is an initial segment of $\leq_{B}$ and $E_{0}<_{B} E$ for any Borel equivalence relation $E$ not in this list.

The linearity of the order $\leq_{B}$ breaks down beyond $E_{0}$. This has been known for some time but it has been amplified more recently by results of Woodin (unpublished), who showed that there are uncountably many pairwise $\leq_{B}$-incomparable Borel equivalence relations, and then by Louveau-Velickovic [25], who showed that the partial order of subsets of the integers under inclusion modulo finite sets embeds into $\leq_{B}$ on Borel equivalence relations. This non-linearity is in fact a crucial point in which Borel cardinality theory differs from the classical Cantor cardinality theory.

We now turn our attention to a particular class of Borel equivalence relations, the so-called countable ones. A Borel equivalence relation $E$ on $X$ is called countable if every equivalence class $[x]_{E}$ is countable. A typical example is the equivalence 
relation induced by a Borel action of a countable group on a standard Borel space, e.g., the one induced by the orbits of a single Borel automorphism. Conversely, a theorem of Feldman-Moore [ 8 asserts that every countable Borel equivalence relation is induced by a Borel action of a countable group. Such equivalence relations have long been studied in ergodic theory and the theory of operator algebras (see, e.g., the surveys [27] and [30]). It also turns out that many equivalence relations, although not necessarily countable, nevertheless are up to $\sim_{B}$ countable, so they fall, for our purposes, in the domain of countable Borel equivalence relations. These include, for example, all the equivalence relations induced by Borel actions of second countable locally compact groups (e.g., Lie groups); see Feldman-Hahn-Moore 7] and Kechris 20. Also the isomorphism relation on various classes of countable models, which in some sense have "finite type", e.g., finitely generated groups, locally finite connected graphs, finite rank torsion-free abelian groups, turn out to be $\sim_{B}$ to countable Borel equivalence relations (see Hjorth-Kechris [16]). There is thus a great variety of interesting examples of (up to $\sim_{B}$ ) countable Borel equivalence relations occurring in many areas of mathematics. See, for example, the papers [19], 22], 23], 24], 5], 18] and the references contained therein, for further information concerning the descriptive study of countable Borel equivalence relations. The facts we are about to state in the next paragraph come from the last paper.

To get some feeling about the general structure of $\leq_{B}$ on countable Borel equivalence relations, we first note that there is a largest one, naturally dubbed universal, and denoted by $E_{\infty}$. Thus $E_{\infty}$ is countable Borel and $E \leq_{B} E_{\infty}$ for every countable Borel $E$. Such an $E_{\infty}$ is uniquely determined up to $\sim_{B}$. Thus, excluding the tame ones (which are those in the list $1,2,3, \cdots, \mathbb{N}, \mathbb{R}$ ), all countable Borel equivalence relations fall in the interval

$$
E_{0} \leq_{B} E \leq_{B} E_{\infty}
$$

(This should be contrasted to the situation concerning general Borel equivalence relations, where, by a result of Friedman-Stanley [9], for every Borel equivalence relation $E$ there is a Borel equivalence relation $F$ with $E<_{B} F$.) It also turns out that there are intermediate ones:

$$
E_{0}<_{B} E<_{B} E_{\infty} .
$$

The first examples were found by applying results of Adams [1], 2], and thus made heavy use of methods and results of ergodic theory. However, rather remarkably, and despite the great plethora of examples of countable Borel equivalence relations, until recently, only two examples of distinct up to $\sim_{B}$ intermediate relations had been found, say $E, F$, and they satisfied $E_{0}<_{B} E<_{B} F<_{B} E_{\infty}$. In particular, it has been a long-standing problem in this theory whether there are incomparable under $\leq_{B}$ countable Borel equivalence relations (see, e.g., Kechris [19]).

The first main result of this paper provides an affirmative answer to this problem and in fact shows that the partial order $\leq_{B}$ on countable Borel equivalence relations is quite complex.

Theorem 1. The partial ordering of Borel sets under inclusion can be embedded in the partial (pre-)order of countable Borel equivalence relations under Borel reducibility. More precisely, there is a map $A \mapsto E_{A}$ assigning to each Borel subset of $2^{\mathbb{N}}, A$, a countable Borel equivalence relation, $E_{A}$, so that

$$
A_{1} \subseteq A_{2} \Leftrightarrow E_{A_{1}} \leq_{B} E_{A_{2}} .
$$


In particular, this shows that every Borel partial ordering can be embedded into $\leq_{B}$ on countable Borel equivalence relations.

Our second main result computes the exact descriptive complexity of the relations $\leq_{B}, \sim_{B}$ on countable Borel equivalence relations. We first fix a parametrization of binary Borel relations on a standard Borel space, which for definiteness we take to be the Baire space $\mathbb{N}^{\mathbb{N}}$. This consists of a $\Pi_{1}^{1}$ (i.e., co-analytic) set $D \subseteq 2^{\mathbb{N}}$ and a map $d \in D \mapsto D_{d}$, assigning to each $d \in D$ a binary Borel relation on $\mathbb{N}^{\mathbb{N}}$ so that every such relation is of the form $D_{d}$ for some $d$. Moreover, $\left(D, d \mapsto D_{d}\right)$ satisfies some natural definability properties spelled out in Section 5 below. Using this, we let

$$
\begin{aligned}
\mathcal{E}_{\leq}= & \left\{(d, e) \in D^{2}: D_{d}, D_{e}\right. \text { are countable Borel equivalence } \\
& \text { relations and } \left.D_{d} \leq_{B} D_{e}\right\}
\end{aligned}
$$

and we similarly define $\mathcal{E}_{\sim}$. A straightforward calculation shows that $\mathcal{E}_{\leq}, \mathcal{E}_{\sim}$ are $\boldsymbol{\Sigma}_{\mathbf{2}}^{\mathbf{1}}$ (= projections of co-analytic sets). The following computes their precise complexities.

Theorem 2. The sets $\mathcal{E}_{\leq}, \mathcal{E}_{\sim}$ are $\boldsymbol{\Sigma}_{\mathbf{2}}^{1}$-complete. In other words, the notions of Borel reducibility and bi-reducibility of countable Borel equivalence relations are $\boldsymbol{\Sigma}_{\mathbf{2}}^{\mathbf{1}}$-complete.

(Recall here that a set $A$ in a standard Borel space $X$ is $\boldsymbol{\Sigma}_{\mathbf{2}}^{\mathbf{1}}$-complete if it is $\boldsymbol{\Sigma}_{\mathbf{2}}^{\mathbf{1}}$ and for any $\boldsymbol{\Sigma}_{\mathbf{2}}^{\mathbf{1}}$ set $B \subseteq Y, Y$ a standard Borel space, there is a Borel function $f: Y \rightarrow X$ with $B=f^{-1}(A)$.)

The proofs of these results depend heavily on the Superrigidity Theorems of Zimmer [35], 5.2.5 and 10.1.6, which deal with reduction of cocycles of measurepreserving actions of linear algebraic groups. In [35] (and other papers referred to in this book) Zimmer also shows, loosely speaking, that, under certain circumstances, the equivalence relation induced by a group action together with (the measure class of) an associated invariant measure "encodes" or "remembers" quite a lot of information concerning the group and the action from which it came. In some sense, the main point in the proofs of the results here is that, as an application of the Zimmer Superrigidity Theory, there is also a "rigidity" phenomenon occurring in the purely descriptive context: Under certain circumstances, quite a bit of information concerning a group $G$ acting on a space $X$ is "encoded" or "remembered" simply by the "Borel cardinality" of the quotient space $X / G$ of the orbits. This "set theoretic rigidity" is undoubtedly an interesting phenomenon that needs to be further explored.

A similar set of ideas is used by Zimmer in studying orbit equivalence. (See Theorem 5.2.1, p. 95 of Zimmer 35].) However, in that application of superrigidity, the cocycles that arise are readily seen, via the Borel Density Theorem, to be Zariski dense. In the situation presented in this paper, we construct cocycles whose algebraic hulls must be analyzed. However, we compensate for this complication by being very careful to arrange things so that the domain groups of our cocycles are, in some sense, incompatible with the target groups.

The organization of this paper is as follows. After an introductory Section 1, we discuss in Section 2 the fundamental notion of cocycle of a group action, and collect together various known facts and techniques concerning this concept that we will need later on. These are scattered in [35] and other publications or they are folklore. It seemed though that it would be helpful to collect them together 
in one place and present them in as detailed and self-contained way as possible, for the convenience of the reader who is not familiar with these kinds of methods. In Section 3, we use the Superrigidity Theorems of Zimmer [35] to derive some cocycle reduction results that we will need in the proofs of our main theorems. Although the presentation here is much more detailed than is customary in a paper written for experts in the ergodic theory of linear algebraic groups, it is still far from self-contained and depends heavily on results from this theory (as well as from the theory of linear algebraic groups that are needed here). At least every attempt has been made to state explicitly the results that are being quoted, and give precise references to standard books, whenever possible. In Section 4, the results in the preceding section are put together to give the proof of Theorem 1 and in Section 5 of Theorem 2.

Section 6 contains an application of these ideas to another problem that has received attention recently, namely the issue of the complexity of the isomorphism problem for torsion-free abelian groups of finite rank. See Hjorth-Kechris [16] and Hjorth [15] for some background on this problem. We denote by $\cong_{n}$ the isomorphism relation on torsion-free abelian groups of rank $\leq n$. These are, up to isomorphism, simply the subgroups of $\left(\mathbb{Q}^{n},+\right)$. We also denote by $\cong_{n}^{*}$ the restriction of $\cong_{n}$ to the rigid groups, i.e., those whose only automorphisms are $x \mapsto \pm x$. There is a classical result of Baer which classifies torsion-free abelian groups of rank 1 . However, it has been a long-standing problem in abelian group theory whether a satisfactory classification can be found for rank 2 or higher groups (see Fuchs [10]). As it is not hard to see that $\cong_{n}$ is $\sim_{B}$ to a countable Borel equivalence relation, one can place all this in the context of the theory of countable Borel equivalence relations and try to compute the complexity of the classification problem for $\cong_{n}$ and $\cong_{n}^{*}$. Baer's result implies that $\left(\cong_{1}\right) \sim_{B}\left(\cong_{1}^{*}\right) \sim_{B} E_{0}$ and clearly $\left(\cong_{n}^{*}\right) \leq_{B}\left(\cong_{n+1}^{*}\right)$, so $E_{0} \leq_{B}\left(\cong_{n}^{*}\right) \leq_{B}\left(\cong_{n+1}^{*}\right) \leq_{B}\left(\cong_{n+1}\right) \leq_{B} E_{\infty}$ for any $n \geq 1$. It has been conjectured in Hjorth-Kechris [16] that for $n \geq 2, \cong_{n}$ is universal, i.e., $\left(\cong_{n}\right) \sim_{B} E_{\infty}$. This is still open but if correct it would quantify the exact complexity of the classification problem of rank 2 or higher torsion-free abelian groups, and would show that in some sense no simple classification is possible. Recently Hjorth [15] has taken a first step in that direction by showing that $E_{0}<_{B}\left(\cong_{n}\right)$ for $n \geq 2$, and Thomas [33] extended this by showing that $E_{0}<_{B}\left(\cong_{n}^{*}\right)$ for $n \geq 2$. Thus the classification problem for rigid rank 2 torsion-free abelian groups is, in some precise sense, definitely more complex than that of the rank 1 case. We prove in Section 6 the following result which shows that for rigid groups the complexity increases with the rank.

Theorem 3. For any $n \geq 1,\left(\cong_{n}^{*}\right)<_{B}\left(\cong_{n+1}^{*}\right)$.

In particular, this shows that $\cong_{n}^{*}$ is not universal for any $n$.

Finally, in Section 7 we discuss some additional facts that can be proved by the methods used in this paper and discuss some open problems.

\section{Preliminaries}

We will use more or less standard notation and terminology concerning group actions and descriptive set theory (see Zimmer [35] and Kechris [21], resp.).

Let $G$ be a locally compact second countable (lcsc) group. By a standard Borel $G$-space we mean a standard Borel space $X$ together with a Borel action $(g, x) \mapsto g \cdot x$ 
of $G$ on $X$. We denote by $E_{G}^{X}$ the corresponding equivalence relation

$$
x E_{G}^{X} y \Leftrightarrow \exists g(g \cdot x=y),
$$

by $G \cdot x$ the orbit of $x$, and by $G_{x}=\{g: g \cdot x=x\}$ the stabilizer of $x$.

For a standard Borel space $X$ (i.e., a Polish space equipped with its Borel structure) by a measure on $X$ we will always mean a probability Borel measure on $X$. Such a measure is non-atomic if $\mu(\{x\})=0, \forall x \in X$. The measures on $X$ also form a standard Borel space denoted by $P(X)$. If $f: X \rightarrow Y$ is Borel and $\mu \in P(X)$, then $f_{*}(\mu) \in P(Y)$ is defined by $f_{*}(\mu)(A)=\mu\left(f^{-1}(A)\right)$. If $X$ is a $G$-space, $\mu \in P(X)$ is invariant if $g \cdot \mu=\mu, \forall g \in G$, and ergodic if every $G$-invariant Borel set $A \subseteq X$ has $\mu$ measure 0 or 1 . This is equivalent to saying that every Borel function $f: X \rightarrow Y, Y$ a standard Borel space, which is $G$-invariant, is constant $\mu$-a.e.

An equivalence relation $E$ on a standard Borel space is called tame (or smooth in the terminology of Zimmer 35]) if there is a Borel function $S: X \rightarrow Y, Y$ a standard Borel space, such that $x E y \Leftrightarrow S(x)=S(y)$. It is of course enough to take $Y=[0,1]$ here. If $E=E_{G}^{X}$, for a standard Borel $G$-space $X$, then this is equivalent to saying that $E$ admits a Borel transversal, i.e., a Borel set meeting every orbit in exactly one point. (This result is even true for $G$ a Polish group (Burgess); see Kechris [21, 18.20 (iii).) Recall that if $G$ is a Polish group and $H$ is a closed subgroup, then the equivalence relation $x E_{H}^{G} y \Leftrightarrow y \in x H$, whose quotient space is the set $G / H$ of left-cosets $h H$ of $H$, is tame (see Kechris [21], 12.17). We call a standard Borel $G$-space $X$ tame if the equivalence relation $E_{G}^{X}$ is tame. In particular, every standard Borel $G$-space $X$ is tame, when $G$ is compact (second countable); see Zimmer [35], 2.1.21.

A Borel equivalence relation on a standard Borel space $X$ is an equivalence relation $E \subseteq X^{2}$ which is a Borel set in $X^{2}$. It is countable if every equivalence class $[x]_{E}, x \in X$, is countable. Every countable Borel equivalence relation $E$ on $X$ is of the form $E=E_{G}^{X}$, for a Borel action of a countable group $G$ on $X$ (FeldmanMoore [8; see also Kechris [21], 18.16). A measure $\mu$ on $X$ is called E-invariant if it is $G$-invariant for any countable $G$ with $E=E_{G}^{X}$ (this is easily seen to be independent of $G$ and the action). It is $E$-ergodic if every $E$-invariant Borel set $A \subseteq X$ has $\mu$-measure 0 or 1 .

Finally, given equivalence relations $E, F$ on standard Borel spaces $X, Y$, resp., we say that $E$ is Borel reducible to $F$, in symbols,

$$
E \leq_{B} F,
$$

if there is a Borel function $f: X \rightarrow Y$ such that

$$
x E y \Leftrightarrow f(x) F f(y) .
$$

We call such an $f$ a reduction of $E$ into $F$. We also let

$$
E \sim_{B} F \Leftrightarrow E \leq_{B} F \text { and } F \leq_{B} E
$$

and

$$
E<_{B} F \Leftrightarrow E \leq_{B} F \text { and } F \not \leq_{B} E .
$$




\section{BASIC FACTS ABOUT COCYCLES}

Let $G$ be a lcsc group, and $X$ a standard Borel $G$-space with invariant probability Borel measure $\mu$. Let $H$ be a lcsc group. A cocycle of this $G$-space into $H$ is a Borel map $\alpha: G \times X \rightarrow H$ such that for all $g, h \in G$

$$
\alpha(h g, x)=\alpha(h, g \cdot x) \alpha(g, x) \quad \mu \text {-a.e. }(x) .
$$

If this equation holds for all $x$ we say that $\alpha$ is a strict cocycle. If $\beta: G \times X \rightarrow H$ is also a cocycle, we say that $\alpha$ is equivalent or cohomologous to $\beta$, in symbols $\alpha \sim \beta$, if there is a Borel map $A: X \rightarrow H$ such that for all $g \in G$,

$$
\alpha(g, x)=A(g \cdot x) \beta(g, x) A(x)^{-1} \quad \mu \text {-a.e. }(x) .
$$

This is clearly an equivalence relation.

For example, suppose that $E=E_{G}^{X}$ and $F=E_{H}^{Z}$, where $H$ acts freely on $Z$. Let $f: X \rightarrow Z$ be a function such that $x E y$ implies $f(x) F f(y)$. Then the function $\alpha: G \times X \rightarrow H$ defined by $f(g \cdot x)=\alpha(g, x) \cdot f(x)$ is a cocycle. Moreover, if $A: X \rightarrow H$ is any Borel function, if we define $f_{0}: X \rightarrow Z$ by $f_{0}(x)=A(x) \cdot f(x)$ and if we define $\alpha_{0}: G \times X \rightarrow H$ by $f_{0}(g \cdot x)=\alpha_{0}(g, x) \cdot f_{0}(x)$, then the cocycle $\alpha_{0}$ is equivalent to $\alpha$; in fact, we have $\alpha_{0}(g, x)=A(g \cdot x) \alpha(g, x)(A(x))^{-1}$.

The main results in this paper are based on various theorems that show that cocycles $\alpha$ as above, under certain circumstances, reduce, i.e., are equivalent to, cocycles $\beta$, whose range $\beta(G \times X)$ is contained in a "small" subgroup of $H$. The following elementary reformulation is useful for this purpose. We need the following terminology, using the above notation: If $H$ acts on some set $Y$, then a function $f: X \rightarrow Y$ will be called $\alpha$-invariant if for all $g \in G$,

$$
\alpha(g, x) \cdot f(x)=f(g \cdot x) \quad \mu \text {-a.e. }(x) .
$$

Also for any closed subgroup $H_{0} \subseteq H$ we denote by $H / H_{0}$ the space of left-cosets $h H_{0}$ of $H_{0}$ and we view $H$ as acting on $H / H_{0}$ by $h_{1} \cdot h H=h_{1} h H$.

We now have:

Proposition 2.1 (see Zimmer [35], 4.2.18(b)). Let $H_{0} \subseteq H$ be a closed subgroup of $H$. Then a cocycle $\alpha: G \times X \rightarrow H$ is equivalent to a cocycle $\beta: G \times X \rightarrow H$ with $\beta(G \times X) \subseteq H_{0}$ iff there exists a Borel $\alpha$-invariant function $f: X \rightarrow H / H_{0}$.

Proof. Say $\alpha(g, x)=A(g \cdot x) \beta(g, x) A(x)^{-1} \mu$-a.e. $(x)$, where $A: X \rightarrow H$ is Borel and $\beta(G \times X) \subseteq H_{0}$. Then if $f(x)=A(x) H_{0}, f: X \rightarrow H / H_{0}$ is Borel and for each $g, \alpha(g, x) \cdot f(x)=f(g \cdot x) \mu$-a.e. $(x)$, as $\beta(g, x) \in H_{0}$. Conversely, given a Borel $\alpha$-invariant $f$, and letting $i: H / H_{0} \rightarrow H$ be a Borel injection such that $i(h H) \in h H$, we let $A(x)=i(f(x))$. Then as $\alpha(g, x) \cdot f(x)=f(g \cdot x) \mu$-a.e. $(x)$, we have that $\alpha(g, x) A(x) \in A(g \cdot x) H_{0} \mu$-a.e. $(x)$, so if $\beta(g, x)=A(g \cdot x)^{-1} \alpha(g, x) A(x)$, then $\beta(g, x) \in H_{0} \mu$-a.e. $(x)$ and $\alpha \sim \beta$. Now we change $\beta(g, x)$ on a Borel set in $G \times X$, whose $G$-sections are $\mu$-null to make sure that $\beta(g, x) \in H_{0}$ for all $g, x$, i.e., $\beta(G \times X) \subseteq H_{0}$.

The next proposition, called the Cocycle Reduction Lemma, gives a convenient tool for showing that cocycles reduce to other ones with small range. Recall that the measure $\mu$ on the standard $G$-space $X$ is called ergodic if every $G$-invariant Borel set has $\mu$-measure 0 or 1 . Also a Borel action of a lcsc group $H$ on a standard Borel space $Y$ is called tame if the corresponding equivalence relation $E_{Y}^{H}$ is tame, i.e., 
there is Borel $S: Y \rightarrow Z, Z$ some Polish space, such that $y_{1} E_{Y}^{H} y_{2} \Leftrightarrow S\left(y_{1}\right)=S\left(y_{2}\right)$. We can of course always take $Z$ to be $[0,1]$ here.

Proposition 2.2 (see Zimmer [35], 5.2.11). Suppose $X$ is a standard Borel Gspace with ergodic, invariant measure $\mu$ and $\alpha: G \times X \rightarrow H$ is a cocycle. Assume also that $Y$ is a tame standard Borel $H$-space. If there is an $\alpha$-invariant Borel function $f: X \rightarrow Y$, then there is $y_{0} \in Y$ such that $\alpha \sim \beta$, for a cocycle $\beta: G \times X \rightarrow H$ with $\beta(G \times X) \subseteq H_{y_{0}}=$ the stabilizer of $y_{0}$. Moreover $f(x) \in H \cdot y_{0}, \mu$-a.e. $(x)$.

Proof. Let $S: Y \rightarrow[0,1]$ be Borel with $y_{1} E_{Y}^{H} y_{2} \Leftrightarrow S\left(y_{1}\right)=S\left(y_{2}\right)$. Let $\hat{f}(x)=$ $S(f(x))$. Then, as $\alpha(g, x) \cdot f(x)=f(g \cdot x) \mu$-a.e. $(x)$, clearly $\hat{f}(g \cdot x)=\hat{f}(x) \mu$-a.e. $(x)$. So $\forall g \forall_{\mu}^{*} x(\hat{f}(g \cdot x)=\hat{f}(x))$ (where $\forall_{\mu}^{*} x P(x) \Leftrightarrow\{x: P(x)\}$ is $\mu$-conull), thus, by Fubini, $\forall_{\mu}^{*} x \forall_{\lambda}^{*} g(\hat{f}(g \cdot x)=\hat{f}(x))$, where $\lambda$ is the Haar measure on $G$. Now the set

$$
X_{0}=\{x: g \mapsto \hat{f}(g \cdot x) \text { is } \lambda \text {-a.e. constant }\}=\left\{x: \forall_{\lambda}^{*} g \forall_{\lambda}^{*} h(\hat{f}(g \cdot x)=\hat{f}(h \cdot x))\right\}
$$

is Borel (see, e.g., Kechris [21, 17.25) and, by the translation invariance of $\lambda, G$ invariant. So if we define $\hat{f}_{0}(x)=$ the value of $\hat{f}(g \cdot x), \lambda$-a.e. $(g)$, if $x \in X_{0}, \hat{f}_{0}(x)=0$, if $x \notin X_{0}$, then clearly $\hat{f}_{0}$ is $G$-invariant and $\hat{f}_{0}=\hat{f}, \mu$-a.e. By ergodicity, it follows that $\hat{f}_{0}$, and thus $\hat{f}$, is constant $\mu$-a.e., and this means that $f$ takes values in a single $H$-orbit, say $H \cdot y_{0}, \mu$-a.e., and we can assume that $f$ actually takes values in $H \cdot y_{0}$ everywhere, by changing $f$ on a set of measure 0 . Now the $H$-action on $H \cdot y_{0}$ can be clearly identified with the $H$-action on $H / H_{y_{0}}$ and thus, since $f$ is an $\alpha$-invariant function, by 2.1 , we have that $\alpha \sim \beta$ with $\beta(G \times X) \subseteq H_{y_{0}}$.

We will next discuss the concepts of induced actions and cocycles.

Suppose $\Gamma \subseteq G$ is a closed subgroup of $G$ and fix a Borel transversal $T$ for the left-cosets of $\Gamma$ with $1 \in T$ (so $T$ meets every such coset in exactly one point). We can clearly identify $T$ with $G / \Gamma$, identifying $t$ with $t \Gamma$. So the action of $G$ on $G / \Gamma$ induces a Borel action of $G$ on $T$ given by

$$
g \cdot t=\text { the unique element of } T \text { in the coset } g t \Gamma .
$$

Also let

$$
\begin{aligned}
\rho(g, t)= & \text { the unique element } \gamma \in \Gamma \\
& \text { such that }(g \cdot t) \gamma=g t \\
= & (g \cdot t)^{-1} g t .
\end{aligned}
$$

Then it is easy to check that $\rho$ is a strict cocycle, $\rho: G \times T \rightarrow \Gamma$. We call this the cocycle associated to $\Gamma$ (and the choice of $T$ ).

Now assume $\Gamma$ acts in a Borel way on a standard Borel space $X$ with invariant measure $\mu$. Assume also that $\Gamma$ is a lattice in $G$, i.e., $\Gamma$ is discrete and the action of $G$ on $G / \Gamma$, and thus equivalently on $T$, admits an invariant probability Borel measure, say $\nu$. We define the induced action of $G$ as follows: Let $Y=X \times T$ and define the action of $G$ on $Y$ by

$$
g \cdot(x, t)=(\rho(g, t) \cdot x, g \cdot t)
$$


It is easy to check, using Fubini, that $\mu \times \nu$ is an invariant measure for this action: If $p: X \times T \rightarrow \mathbb{R}$ is bounded Borel, then

$$
\begin{aligned}
\iint(g \cdot p) d(\mu \times \nu) & =\int\left(\int p(g \cdot(x, t)) d \mu(x)\right) d \nu(t) \\
& \left.=\int\left(\int p(\rho(g, t) \cdot x, g \cdot t)\right) d \mu(x)\right) d \nu(t) \\
& =\int\left(\int p(x, g \cdot t) d \mu(x)\right) d \nu(t) \\
& =\int\left(\int p(x, g \cdot t) d \nu(t)\right) d \mu(x),
\end{aligned}
$$

so

$$
\begin{aligned}
\iint(g \cdot p) d(\mu \times \nu) & =\int\left(\int p(g \cdot(x, t)) d \mu(x)\right) d \nu(t) \\
& =\int\left(\int p(x, t) d \nu(t)\right) d \mu(x) \\
& =\iint p d(\mu \times \nu) .
\end{aligned}
$$

Next we check that if $\mu$ is ergodic, so is $\mu \times \nu$. Indeed, let $A \subseteq X \times T$ be Borel $G$-invariant, and put $\left.A^{t}=\{x:(x, t) \in A)\right\}$. Then $\rho(g, t) \cdot A^{t}=A^{g \cdot t}$ and $A^{1}$ is $\Gamma$-invariant, so $\mu\left(A^{1}\right)=0$ or 1 , thus, since $\rho(g, 1) \cdot A^{1}=A^{g \cdot 1}$, all $A^{t}$ have the same $\mu$-measure as $A^{1}$, so $(\mu \times \nu)(A)=0$ or 1 .

Now given any strict cocycle $\alpha: \Gamma \times X \rightarrow H$ we define the strict cocycle $\beta$ : $G \times Y \rightarrow H$ by

$$
\beta(g,(x, t))=\alpha(\rho(g, t), x),
$$

and call this the induced cocycle.

For example, suppose that $E=E_{\Gamma}^{X}$ and $F=E_{H}^{Z}$, where $H$ acts freely on $Z$. Let $f: X \rightarrow Z$ be a function such that $x E x^{\prime}$ implies $f(x) F f\left(x^{\prime}\right)$. Let $\alpha: \Gamma \times X \rightarrow H$ be the cocycle defined by $f(\gamma \cdot x)=\alpha(\gamma, x) \cdot f(x)$. Let $G$ act on $Y=X \times T$ by $g \cdot(x, t)=(\rho(g, t) \cdot x, g \cdot x)$. Define $f_{0}: Y \rightarrow Z$ by $f_{0}(x, t)=f(x)$. Let $E_{0}=E_{G}^{Y}$. Then $y E_{0} y^{\prime}$ implies $f_{0}(y) F f_{0}\left(y^{\prime}\right)$. Let $\alpha_{0}: G \times Y \rightarrow H$ be the cocycle defined by $f_{0}(g \cdot y)=\alpha_{0}(g, y) \cdot f_{0}(y)$. Then $\alpha_{0}$ is induced from $\alpha$; that is, we have $\alpha_{0}(g,(x, t))=\alpha(\rho(g, t), x)$.

We have the following fact.

Proposition 2.3. Suppose that $\Gamma$ is a lattice in $G$, that $X$ is a standard Borel $\Gamma$-space with invariant measure $\mu$, that $\alpha: \Gamma \times X \rightarrow H$ is a strict cocycle and that $\beta: G \times Y \rightarrow H$ is the induced cocycle for the induced $G$-space $Y$. Assume that $H_{0}$ is a closed subgroup of $H$ and that there is $\beta_{1}: G \times Y \rightarrow H$ with $\beta_{1} \sim \beta$ and with $\beta_{1}(G \times Y) \subseteq H_{0}$. Then there is $\alpha_{1}: \Gamma \times X \rightarrow H$ with $\alpha_{1} \sim \alpha$ and $\alpha_{1}(\Gamma \times X) \subseteq H_{0}$.

Proof. By 2.1, there is a $\beta$-invariant Borel function $f: Y=(X \times T) \rightarrow H / H_{0}$, i.e., for all $g \in G$,

$$
\beta(g,(x, t)) \cdot f(x, t)=f(g \cdot(x, t))(\mu \times \nu) \text {-a.e. }(x, t) .
$$

Let $U$ be the space of all Borel functions from $X$ into $H / H_{0}$, two functions being identified if they agree $\mu$-a.e. Consider then the map $\Phi: T \rightarrow U$ given by $\Phi(t)(x)=$ 
$f(x, t)$. Let $\Gamma$ act on $U$ by

$$
\gamma \cdot p(x)=\alpha\left(\gamma, \gamma^{-1} \cdot x\right) \cdot p\left(\gamma^{-1} \cdot x\right)
$$

We then claim that $\Phi$ is $\rho$-invariant, i.e., for any $g \in G$,

$$
\rho(g, t) \cdot \Phi(t)=\Phi(g \cdot t) \quad \nu \text {-a.e. }(t) .
$$

Indeed,

$$
\begin{aligned}
(\rho(g, t) \cdot \Phi(t))(x) & =\alpha\left(\rho(g, t), \rho(g, t)^{-1} \cdot x\right) \cdot \Phi(t)\left(\rho(g, t)^{-1} \cdot x\right) \\
& =\alpha\left(\rho(g, t), \rho(g, t)^{-1} \cdot x\right) \cdot f\left(\rho(g, t)^{-1} \cdot x, t\right) .
\end{aligned}
$$

Now

$$
\begin{aligned}
\beta(g,(x, t)) \cdot f(x, t) & =f(g \cdot(x, t)) \\
& =f(\rho(g, t) \cdot x, g \cdot t)(\mu \times \nu)-a . e \cdot(x, t),
\end{aligned}
$$

so

$$
\alpha(\rho(g, t), x) \cdot f(x, t)=f(\rho(g, t) \cdot x, g \cdot t)(\mu \times \nu)-a . e .(x, t) .
$$

Thus for $\nu$-a.e. $(t)$ and using the $\Gamma$-invariance of $\mu$ to substitute $\rho(g, t)^{-1} \cdot x$ for $x$, we have

$$
\begin{aligned}
f(x, g \cdot t) & =\alpha\left(\rho(g, t), \rho(g, t)^{-1} \cdot x\right) \cdot f\left(\rho(g, t)^{-1} \cdot x, t\right) \\
& =(\rho(g, t) \cdot \Phi(t))(x) \quad \mu-a . e \cdot(x)
\end{aligned}
$$

and, since

$$
f(x, g \cdot t)=\Phi(g \cdot t)(x)
$$

this shows that

$$
\rho(g, t) \cdot \Phi(t)=\Phi(g \cdot t) \quad \nu \text {-a.e. }(t) .
$$

We now claim that this implies that there is a fixed point $p \in U$ for the $\Gamma$-action, i.e., $\gamma \cdot p=p$ for all $\gamma \in \Gamma$. Granting this we have for any $\gamma \in \Gamma$,

$$
\begin{aligned}
\gamma \cdot p(x) & =\alpha\left(\gamma, \gamma^{-1} \cdot x\right) \cdot p\left(\gamma^{-1} \cdot x\right) \\
& =p(x) \quad \mu \text {-a.e. }(x),
\end{aligned}
$$

so putting $\gamma \cdot x$ for $x$ and using again the $\Gamma$-invariance of $\mu$, we have

$$
p(\gamma \cdot x)=\alpha(\gamma, x) \cdot p(x) \quad \mu-a . e .(x),
$$

i.e., $p$ is an $\alpha$-invariant function from $X$ into $H / H_{0}$, so by $2.1, \alpha \sim \alpha_{1}$, for some cocycle $\alpha_{1}: \Gamma \times X \rightarrow H$ with $\alpha_{1}(\Gamma \times X) \subseteq H_{0}$.

To prove the existence of such a fixed point $p$ we argue as follows (see Zimmer 35], 4.2.19): Let $\sigma: G \rightarrow \Gamma$ be defined by $\sigma(g)=\rho(g, 1)$. Then notice that for any $g \in G, t \in T, \sigma(g t)=\rho(g t, 1)=\rho(g, t \cdot 1) \rho(t, 1)=\rho(g, t) \sigma(t)$, so $\rho(g, t)=$ $\sigma(g t) \sigma(t)^{-1}$. It follows that for each $g \in G$,

$$
\sigma(g t)^{-1} \cdot \Phi(g \cdot t)=\sigma(t)^{-1} \cdot \Phi(t) \quad \nu \text {-a.e. }(t) .
$$

So, by Fubini, there is $t_{0} \in T$ such that for almost all $g \in G$ (with respect to the Haar measure), $\sigma\left(g t_{0}\right)^{-1} \cdot \Phi\left(g \cdot t_{0}\right)=\sigma\left(t_{0}\right)^{-1} \cdot \Phi\left(t_{0}\right)$. Put $p=\sigma\left(t_{0}\right)^{-1} \cdot \Phi\left(t_{0}\right)$. Then viewing now $\Phi$ as defined on $G / \Gamma$ by identifying $t \in T$ with $t \Gamma \in G / \Gamma$, and using the translation invariance of the Haar measure, we conclude that for almost all $g \in G, \sigma(g)^{-1} \cdot \Phi(g \Gamma)=p$. Now notice that for $\gamma \in \Gamma, \sigma(g \gamma)=\sigma(g) \gamma$, so $\gamma^{-1} \cdot p=\gamma^{-1} \sigma(g)^{-1} \cdot \Phi(g \Gamma)=\sigma(g \gamma)^{-1} \cdot \Phi(g \Gamma)=\sigma(g \gamma)^{-1} \cdot \Phi(g \gamma \Gamma)$, for almost all $g \in G$, so, again by translation invariance, $\gamma^{-1} \cdot p=p$ and we are done. 
Proposition 2.4. Suppose $H$ is lcsc and $H_{1} \subseteq H$ is a closed subgroup. Suppose $G$ acts on $X$ with invariant, ergodic measure $\mu$ and $\alpha: G \times X \rightarrow H_{1}$ is a Borel cocycle. Assume that viewing $\alpha$ as a cocycle into $H, \alpha: G \times X \rightarrow H$, we have a cocycle $\beta: G \times X \rightarrow H$ with $\beta \sim \alpha$ and $\beta(G \times X) \subseteq H_{0}$, where $H_{0}$ is a closed subgroup of $H$. If the action of $H_{0}$ on $H / H_{1}$ is tame, then there is $h \in H$ and a cocycle $\alpha_{1}: G \times X \rightarrow H_{1}$ such that $\alpha \sim \alpha_{1}$ and $\alpha_{1}(G \times X) \subseteq H_{1} \cap h H_{0} h^{-1}$.

In particular, this conclusion holds if $H_{0}$ is compact or if $H_{0}$ is arbitrary (closed) and $H_{1}$ has finite index in $H$.

Proof. By assumption and 2.1 there is an $\alpha$-invariant Borel function $f: X \rightarrow H / H_{0}$. Now we claim that the action of $H_{1}$ on $H / H_{0}$ is tame. Granting this, we have, by 2.2 , that there is $h H_{0} \in H / H_{0}$ such that $\alpha$ is equivalent (as a cocycle into $H_{1}$ ) to a cocycle taking values in the stabilizer of $h H_{0}$ in the action by $H_{1}$. This stabilizer is the set $\left\{h_{1} \in H_{1}: h_{1} \cdot h H_{0}=h H_{0}\right\}=H_{1} \cap h H_{0} h^{-1}$.

To prove the claim, notice that there is a canonical bijection between the double cosets $H_{1} h H_{0}, h \in H$, of $\left(H_{1}, H_{0}\right)$ and the orbits of the $H_{1}$ action on $H / H_{0}$, namely $H_{1} h H_{0} \mapsto H_{1} \cdot\left(h H_{0}\right)$. Thus the action of $H_{1}$ on $H / H_{0}$ is tame iff the equivalence relation induced by the double cosets is tame. Clearly the equivalence relation induced by the double cosets $H_{1} h H_{0}$ is tame iff the equivalence relation induced by the double cosets $H_{0} h H_{1}$ is tame (using $x \mapsto x^{-1}$ ) iff the action of $H_{0}$ into $H / H_{1}$ is tame, and this last statement is assumed in our hypothesis.

Finally, notice that if $H_{0}$ is compact or $H_{1}$ has finite index in $H$, clearly $H_{0}$ acts tamely on $H / H_{1}$.

Suppose again that $G$ acts on $X$ preserving a measure $\mu$. By a finite extension of this action we mean a standard Borel $G$-space $\hat{X}$ with an invariant measure $\hat{\mu}$, together with a Borel map $\pi: \hat{X} \rightarrow X$ such that

(i) for all $g \in G$ and for all $x \in \hat{X}$, we have $\pi(g \cdot x)=g \cdot \pi(x)$,

(ii) $\pi$ is finite-to- 1 ,

(iii) $\pi_{*}(\hat{\mu})=\mu$.

If $\alpha: G \times X \rightarrow H$ is a cocycle, then its lift to $\hat{X}, \hat{\alpha}: G \times \hat{X} \rightarrow H$, is defined by $\hat{\alpha}(g, \hat{x})=\alpha(g, \pi(\hat{x}))$.

The argument in the next proposition is similar to that of the proof of [35], 9.2.6.

Proposition 2.5. Suppose that $X$ is a standard Borel G-space with ergodic, invariant measure $\mu$. Let $\alpha: G \times X \rightarrow H$ be a Borel cocycle. Suppose $H_{0} \subseteq H$ is a closed subgroup of $H$ with finite index. Then there exists a finite extension $\hat{X}$ of $X$ which is also ergodic such that the lift $\hat{\alpha}$ of $\alpha$ to $\hat{X}, \hat{\alpha}: G \times \hat{X} \rightarrow H$, is equivalent to a cocycle taking values in $H_{0}$.

Proof. Let $H_{1}$ be a minimal closed subgroup of $H$ satisfying $H_{0} \subseteq H_{1} \subseteq H$ such that $\alpha$ is equivalent to a cocycle $\beta$ taking values into $H_{1}$. Then $\beta$ has the property that no cocycle equivalent to it takes values into a proper closed subgroup of $H_{1}$ containing $H_{0}$. So by replacing $\alpha$ by $\beta$ if necessary, we can assume that $\alpha$ is not equivalent to a cocycle taking values into a proper closed subgroup of $H$ containing $H_{0}$, and show how to find an ergodic finite extension $\hat{X}$ of $X$ so that the lift $\hat{\alpha}$ of $\alpha$ is equivalent to a cocycle taking values in $H_{0}$.

Let $\hat{X}=X \times\left(H / H_{0}\right)$ and define the action of $G$ on $\hat{X}$ by

$$
g \cdot\left(x, h H_{0}\right)=\left(g \cdot x, \alpha(g, x) h H_{0}\right)
$$


and let $\pi\left(x, h H_{0}\right)=x$. We give $\hat{X}$ the product measure $\mu \times \nu$, where $\nu$ is the counting measure on $H / H_{0}$. Clearly this is a finite extension of $X$. Consider the second projection map $\rho\left(x, h H_{0}\right)=h H_{0}, \rho: \hat{X} \rightarrow H / H_{0}$. It is clearly $\hat{\alpha}$-invariant, so by $2.1, \hat{\alpha}$ is equivalent to a cocycle taking values into $H_{0}$.

So it only remains to show that $\mu \times \nu$ is ergodic. Assume $A \subseteq \hat{X}$ is a $G$ invariant Borel set and $(\mu \times \nu)(A)>0$, in order to show that $(\mu \times \nu)(A)=1$. Then $\mu(\pi(A))>0$, so since $\pi(A)$ is a $G$-invariant Borel subset of $X$, we have, by ergodicity in $X$, that $\mu(\pi(A))=1$. Put $B=\pi(A)$, and letting $F=$ power set of $H / H_{0}$, define $f: X \rightarrow F$ by

$$
f(x)=\left\{\begin{array}{l}
\rho\left(\pi^{-1}(x) \cap A\right), \text { if } x \in B, \\
\emptyset, \text { otherwise. }
\end{array}\right.
$$

Then considering the obvious action of $H$ on $F$ (induced by its action on $H / H_{0}$ ) we see that $f$ is $\alpha$-invariant; so by 2.2 there is $s_{0} \in F$ such that $f(x) \in H \cdot s_{0} \mu$-a.e. $(x)$, and there is $\beta$ with $\alpha \sim \beta$ and $\beta$ taking values in the stabilizer (in $H$ ) of $s_{0}$, say $H_{1}$. If $s_{0} \neq H / H_{0}$, then clearly $H_{1}$ is a closed proper subgroup of $H$ with $H_{0} \subseteq H_{1}$, a contradiction. So $s_{0}=H / H_{0}$ and $f(x)=H / H_{0} \mu$-a.e. $(x)$, thus $\nu\left(A_{x}\right)=1 \mu$-a.e. $(x)$, so $(\mu \times \nu)(A)=1$.

Proposition 2.6. Suppose that $X$ is a standard Borel $G$-space with ergodic, invariant measure $\mu$. Let $\alpha: G \times X \rightarrow H$ be a cocycle. Let $\hat{X}$ be a finite extension of $X$ and $\hat{\alpha}: G \times \hat{X} \rightarrow H$ the lift of $\alpha$. If $\hat{\alpha}$ is equivalent to a cocycle taking values in a compact subgroup of $H$, then $\alpha$ is equivalent to a cocycle taking values in a compact subgroup of $H$.

Proof. Say $\hat{\alpha} \sim \hat{\beta}$ with $\hat{\beta}(G \times \hat{X}) \subseteq K, K$ a compact subgroup of $H$. Then, by 2.1 there is a Borel map $\hat{f}: \hat{X} \rightarrow H / K$ which is $\hat{\alpha}$-invariant. Let $F$ be the standard Borel space of non-empty finite subsets of $H / K$ and let $f: X \rightarrow F$ be defined by $f(x)=\hat{f}\left(\pi^{-1}(x)\right)$. Then it is easy to check that $f$ is $\alpha$-invariant. By considering the obvious action of $H$ on $F$ (induced by the action of $H$ on $H / K$ ), we conclude, using 2.2, that, if this action is tame, then $\alpha$ is equivalent to a cocycle taking values into the stabilizer of some point for the action of $H$ on $F$, which is clearly compact.

To see that this action is tame, it is enough to check, for each $n$, that the action of $H$ on the subsets of $H / K$ of cardinality $n$ is tame, and this is the same as checking that the action of $H \times S_{n}\left(S_{n}=\right.$ the symmetric group in $n$ elements) on the space of distinct $n$-tuples $\left\langle a_{1}, \cdots, a_{n}\right\rangle$ from $H / K$ is tame, where for $(h, \rho) \in H \times S_{n}$, $(h, \rho) \cdot\left\langle a_{1}, \cdots, a_{n}\right\rangle=\left\langle h \cdot a_{\rho(1)}, \cdots, h \cdot a_{\rho(n)}\right\rangle$. Since $H$ has finite index in $H \times S_{n}$, it is enough to check that the action of $H$ on the space of distinct $n$-tuples is tame. Call $E$ the corresponding equivalence relation. Now the action of $K$ on the space of distinct $n$-tuples is tame, as $K$ is compact. Denote by $E^{\prime}$ its corresponding equivalence relation. Let $\vartheta: H / K \rightarrow H$ be Borel such that $\vartheta(h K) \cdot h K=K$. Then notice that $\left\langle a_{1}, \cdots, a_{n}\right\rangle E\left\langle b_{1}, \cdots, b_{n}\right\rangle$ iff $\vartheta\left(a_{1}\right) \cdot\left\langle a_{1}, \cdots, a_{n}\right\rangle E \vartheta\left(b_{1}\right) \cdot\left\langle b_{1}, \cdots, b_{n}\right\rangle$ iff $\vartheta\left(a_{1}\right) \cdot\left\langle a_{1}, \cdots, a_{n}\right\rangle E^{\prime} \vartheta\left(b_{1}\right) \cdot\left\langle b_{1}, \cdots, b_{n}\right\rangle$, so $E$ is tame as well.

Here is the final fact that we will need.

Proposition 2.7. Suppose $X$ is a standard Borel G-space with ergodic, invariant measure and $\alpha: G \times X \rightarrow H$ is a cocycle. Let $\pi: G^{\prime} \rightarrow G$ be a continuous homomorphism such that $\pi\left(G^{\prime}\right)$ has finite index in $G$. Let $G^{\prime}$ act on $X$ by $g^{\prime} \cdot x=$ $\pi\left(g^{\prime}\right) \cdot x$ and let $\alpha^{\prime}: G^{\prime} \times X \rightarrow H$ be given by $\alpha^{\prime}\left(g^{\prime}, x\right)=\alpha\left(\pi\left(g^{\prime}\right), x\right)$. Assume that 
$\alpha^{\prime}$ is equivalent to a cocycle taking values in a compact subgroup of $H$. Then $\alpha$ is equivalent to a cocycle taking values in a compact subgroup of $H$.

Proof. Suppose $\alpha^{\prime} \sim \beta^{\prime}$ with $\beta^{\prime}\left(G^{\prime} \times X\right) \subseteq K, K$ a compact subgroup of $H$. Then, by 2.1 , there is an $\alpha^{\prime}$-invariant map $f: X \rightarrow H / K$. As in the proof of 2.3 , let $U$ be the space of all Borel maps from $X$ into $H / K$, two functions being identified if they agree a.e. Let $G$ act on $U$ by

$$
g \cdot p(x)=\alpha\left(g, g^{-1} \cdot x\right) \cdot p\left(g^{-1} \cdot x\right) .
$$

Then $f$ is $\pi\left(G^{\prime}\right)$-invariant, so the orbit of $f$ under $G$ is finite, say equal to the set $\left\{f_{0}, f_{1}, \cdots, f_{n-1}\right\}$. Then let $f^{*}$ be the Borel map from $X$ into the space $F$ of non-empty finite subsets of $H / K$, defined by $f^{*}(x)=\left\{f_{0}(x), \cdots, f_{n-1}(x)\right\}$. Then if we let for $g \in G, g \cdot f^{*}(x)=\left\{g \cdot f_{0}(x), \cdots, g \cdot f_{n-1}(x)\right\}, g \cdot f^{*}=f^{*}$ a.e., that is, $g \cdot f^{*}(x)=\alpha\left(g, g^{-1} \cdot x\right) \cdot f^{*}\left(g^{-1} \cdot x\right)=f^{*}(x)$, a.e. $(x)$, thus $\alpha(g, x) \cdot f^{*}(x)=$ $f^{*}(g \cdot x)$, a.e. $(x)$, which means that $f^{*}$ is $\alpha$-invariant, where we view $H$ as acting on $F$ by the action induced from the action of $H$ on $H / K$. Now the action of $H$ on $F$ is tame, by the proof in 2.6, and has compact stabilizers, so, by $2.2, \alpha$ is equivalent to a cocycle taking values in a compact subgroup of $H$.

\section{Cocycle Reduction Results}

Recall that a lcsc group $G$ is amenable if there is a (left)-invariant mean on $L^{\infty}(G)\left(=L^{\infty}(G, \lambda)\right.$, where $\lambda$ is the Haar measure on $\left.G\right)$, i.e., a normalized $(\Lambda(1)=$ 1) positive linear functional $\Lambda \in\left(L^{\infty}(G)\right)^{*}$, such that $\Lambda(f)=\Lambda\left(f_{g}\right)$, where $f_{g}(h)=$ $f(g h)$.

Also a lcsc group $G$ is called a Kazhdan group (or a group with property (T)) if for any unitary representation $\pi: G \rightarrow U(H)$, where $U(H)$ is the unitary group of a separable Hilbert space $H$, if $\pi$ almost has invariant vectors (i.e., for every $\epsilon>0$ and every compact $K \subseteq G$ there is a unit vector $v \in H$ such that $\|\pi(g)(v)-v\|<$ $\epsilon, \forall g \in K)$, then $\pi$ has a non-0 invariant vector.

Theorem 3.1 (Zimmer 35], 9.1.1). Let $G$ be a Kazhdan group and $X$ a standard Borel $G$-space with an invariant, ergodic measure. Let $H$ be an amenable group. Then for every cocycle $\alpha: G \times X \rightarrow H$ there is an equivalent cocycle $\beta: G \times X \rightarrow H$ and a compact subgroup $K \subseteq H$ such that $\beta(G \times X) \subseteq K$.

Theorem 3.2 (Zimmer [36], 2.2). Let $G$ be a Kazhdan group and $X$ a standard Borel $G$-space with an ergodic, invariant measure. Let $\Gamma$ be a countable group. Then for every cocycle $\alpha: G \times X \rightarrow \Gamma$ there is an equivalent cocycle $\beta: G \times X \rightarrow \Gamma$ and a finitely generated subgroup $\Gamma_{0} \subseteq \Gamma$ with $\beta(G \times X) \subseteq \Gamma_{0}$.

Next we will review some cocycle reduction results for actions of algebraic groups. We will adapt the terminology and (with some minor exceptions) the notation of [35], Chapter 3. In particular, we take $\Omega$ to be an algebraically closed field of characteristic 0 containing $\mathbb{R}$ and all p-adic fields $\mathbb{Q}_{p}, p$ a prime. Then an algebraic group $G$ is a subgroup of some $\mathrm{GL}_{n}(\Omega)$ (= the group of invertible $n \times n$ matrices over $\Omega$ ), which is Zariski closed in $\mathrm{GL}_{n}(\Omega)$, i.e., consists of all matrices $M$ in $\operatorname{GL}_{n}(\Omega)$ which satisfy a set of equations $f_{1}(M)=0, \cdots, f_{k}(M)=0$, where each $f_{i}$ is a polynomial in $\Omega\left[x_{1}, \cdots, x_{n^{2}}\right]$ (we thus view $M$ as a member of $\Omega^{n^{2}}$ ). If the equations defining $G$ have coefficients in a subfield $k \subseteq \Omega$, then we call $G$ a 
$k$-group. For any subring $A \subseteq \Omega$, we let $\mathrm{GL}_{n}(A)=\left\{\left(a_{i j}\right) \in \mathrm{GL}_{n}(\Omega): a_{i j} \in A\right.$ and $\left.\left(\operatorname{det}\left(a_{i j}\right)\right)^{-1} \in A\right\}$, and define for any algebraic group $G \subseteq \mathrm{GL}_{n}(\Omega)$,

$$
G(A)=G \cap \mathrm{GL}_{n}(A) .
$$

In particular, if $G$ is a $k$-group and $A=k, G(k)$ is the group of all matrices in $G$ with coefficients in $k$.

The topology of an algebraic group $G$ is always the Zariski topology on $G$. When $G$ is a $k$-group and $k$ is one of the fields $\mathbb{R}, \mathbb{C}, \mathbb{Q}_{p}$, then $G(k)$, as a group of matrices over these fields, is a lcsc group with the topology it inherits from $k^{n^{2}}$. So any topological notions about the group $G(k)$ refer to this topology (sometimes called the Hausdorff topology to distinguish it from the Zariski topology).

The proofs of our main results are based on the superrigidity theorems of Zimmer [35. We only state the particular instances of these results that we need.

The first result is a special case of [35], 10.1.6, and we use the context of Chapter 10 in that book.

Theorem 3.3 (Zimmer [35], 10.1.6). Suppose $p, q$ are distinct rational primes and let $G$ be a connected semisimple $\mathbb{Q}_{p}$-group which is moreover algebraically simply connected, almost $\mathbb{Q}_{p}$-simple and satisfies $\mathbb{Q}_{p}-\operatorname{rank}(G) \geq 2$. Let $H$ be a connected $\mathbb{Q}_{q}$-simple, $\mathbb{Q}_{q}$-group. Assume that $X$ is a standard Borel $G\left(\mathbb{Q}_{p}\right)$-space, with invariant, ergodic measure, and that $\alpha: G\left(\mathbb{Q}_{p}\right) \times X \rightarrow H\left(\mathbb{Q}_{q}\right)$ is a cocycle. For every proper $\mathbb{Q}_{q}$-subgroup $L$ of $H$, assume that $\alpha$ is not equivalent to any cocycle with range contained in $L\left(\mathbb{Q}_{q}\right)$. Then $\alpha$ is equivalent to a cocycle with range contained in a compact subgroup of $H\left(\mathbb{Q}_{q}\right)$.

We will briefly comment on why this is a special case of Zimmer [35], 10.1.6. We take $S=\{p\}$ in that theorem, so that our $G$ is $G_{p}$ in his notation, and $\operatorname{rank}(G)=$ $\mathbb{Q}_{p}-\operatorname{rank}(G)$ (see top of page 189 in [35]-note however that what he calls $G$ is our $G\left(\mathbb{Q}_{p}\right)$ ). Then by the first paragraph of Chapter 10, page 187 of this book, the hypothesis that $\mathbb{Q}_{p}-\operatorname{rank}(G) \geq 2$ implies that $G\left(\mathbb{Q}_{p}\right)$ is not compact and thus has no compact factors, i.e., there is no proper normal $\mathbb{Q}_{p}$-subgroup $H \subseteq G$ with $G\left(\mathbb{Q}_{p}\right) / H\left(\mathbb{Q}_{p}\right)$ compact, since the hypothesis of almost $\mathbb{Q}_{p}$-simplicity means exactly that every such $H$ is finite. Finally, we have to check that $X$ is an irreducible $G\left(\mathbb{Q}_{p}\right)$ space, which according to the definition used in this context in page 188, line 5 in 35] (with the word "proper" omitted in this line, being a typo), this reduces in our case to the fact that the action is ergodic, since almost $\mathbb{Q}_{p}$-simplicity means exactly that there are no infinite normal $\mathbb{Q}_{p}$-subgroups of $G$. Thus all the hypotheses of 10.1.6 are satisfied. Then since, in the notation of 10.1.6, $k=\mathbb{Q}_{q}$ in our case with $q \neq p,(\mathrm{~b}),(\mathrm{c})$ of this theorem do not apply, thus by (a), $\alpha$ is equivalent to a cocycle with values in a compact subgroup of $H\left(\mathbb{Q}_{q}\right)$.

The second and final superrigidity result that we will need is the following, which is a special case of [35], 5.2.5.

Theorem 3.4 (Zimmer [35], 5.2.5). Let $G$ be a connected, semisimple $\mathbb{R}$-group and assume that $G(\mathbb{R})$ is connected (in the Hausdorff topology), that every normal subgroup of $G(\mathbb{R})$ is contained in its center which is finite, and that $\mathbb{R}-\operatorname{rank}(G) \geq 2$. Let $X$ be a standard Borel $G$-space with invariant, ergodic measure. Let $H$ be a connected $k$-simple, $k$-group, where $k=\mathbb{Q}_{p}$ or $\mathbb{R}$. If $\alpha: G(\mathbb{R}) \times X \rightarrow H(k)$ is a cocycle such that $\alpha$ is not equivalent to a cocycle with range contained in a subgroup 
of $H(k)$ of the form $L(k)$, for a proper $k$-subgroup $L$ of $H$, then either $\alpha$ is equivalent to a cocycle taking values into a compact subgroup of $H(k)$ or else $k=\mathbb{R}$ and there is a non-trivial $\mathbb{R}$-morphism from $G$ into $H$.

We will again briefly comment on why this is a special case of [35, 5.2.5. Since $G(\mathbb{R})$ is connected, we have that $G_{\mathbb{R}}^{0}$ in Zimmer's notation, which is the connected component of the identity in $G(\mathbb{R})$ with respect to the Hausdorff topology (see page 35, paragraph 4), is the same as $G(\mathbb{R})$. The irreducibility condition (see [35, 2.2.11) becomes just ergodicity, since every normal subgroup of $G(\mathbb{R})$ is assumed to be contained in the center. So we must have (i) or (ii) in 5.2.5. If $k=\mathbb{R}$ and $H(k)$ is compact, there is nothing to prove. Otherwise, since $H$ is assumed to be $k$-simple we have a $k$-morphism $\pi: G \rightarrow H$, such that $\alpha$ is equivalent to the cocycle $\alpha_{0}(g, x)=\pi(g)$. If $\pi$ is non-trivial we are done; otherwise $\alpha_{0}$ obviously takes values in $\{1\}$, which is compact. If $k=\mathbb{Q}_{p}$, then (iii) applies and we are also done.

We are now ready to derive a cocycle reduction result that we will need in Section 4 .

Below let $F$ be the quadratic form defined by

$$
F\left(x_{1}, x_{2}, \cdots, x_{7}\right)=x_{1}^{2}+\cdots+x_{7}^{2}
$$

and let $\mathrm{SO}_{F}$ be the $\mathbb{Q}$-group (thus $\mathbb{Q}_{p}$-group) of all $7 \times 7$ matrices preserving $F$ and having determinant 1 , that is, all $7 \times 7$ matrices $A$ with $\operatorname{det}(A)=1, A A^{t}=I$.

Note that $\mathrm{SO}_{F}(\mathbb{R})$ is compact. Consequently, for any set $S$ of primes, the group $\Gamma_{S}=\mathrm{SO}_{F}\left(\mathbb{Z}\left[S^{-1}\right]\right)$ may be thought of as a lattice in $\prod_{p \in S} \mathrm{SO}_{F}\left(\mathbb{Q}_{p}\right)$. In the sequel, we will be using induction of cocycles together with 10.1.6 of [35] to show that, if $S$ and $T$ are sets of primes and if $S \neq T$, then a finite measure preserving action of $\Gamma_{S}$ cannot be reducible to a free finite measure preserving action of $\Gamma_{T}$.

Fix an integer $n \geq 3$. Note, for all primes $p$, that $\Gamma_{p}^{\prime}=\mathrm{SL}_{n}(\mathbb{Z}[1 / p])$ is a lattice in $\mathrm{SL}_{n}(\mathbb{R}) \times \mathrm{SL}_{n}\left(\mathbb{Q}_{p}\right)$; moreover, if we project $\Gamma_{p}^{\prime}$ into $\mathrm{SL}_{n}\left(\mathbb{Q}_{p}\right)$, it becomes a dense subgroup, and is not a lattice. So, if $p$ and $q$ are distinct primes, then it will be difficult to use induction of cocycles and 10.1.6 of [35] to show that a finite measure preserving action of $\Gamma_{p}^{\prime}$ cannot be reducible to a free finite measure preserving action of $\Gamma_{q}^{\prime}$. The problem is that the ambient groups are $\mathrm{SL}_{n}(\mathbb{R}) \times \mathrm{SL}_{n}\left(\mathbb{Q}_{p}\right)$ and $\mathrm{SL}_{n}(\mathbb{R}) \times \mathrm{SL}_{n}\left(\mathbb{Q}_{q}\right)$; these two groups have a common factor, making 10.1.6 of [35] difficult to apply. It may be possible to surmount this difficulty, but we avoid it entirely by using $\mathrm{SO}_{F}$ in place of $\mathrm{SL}_{n}$.

We have the following cocycle reduction result.

Theorem 3.5. Let $p$ and $q$ be distinct rational primes. Let $X$ be a standard Borel $\mathrm{SO}_{F}\left(\mathbb{Q}_{p}\right)$-space with an invariant, ergodic probability measure. Let $\alpha: \mathrm{SO}_{F}\left(\mathbb{Q}_{p}\right) \times$ $X \rightarrow \mathrm{SO}_{F}\left(\mathbb{Q}_{q}\right)$ be a cocycle. Then $\alpha$ is equivalent to a cocycle taking values in a compact subgroup of $\mathrm{SO}_{F}\left(\mathbb{Q}_{q}\right)$.

Proof. We will appropriately apply 3.3. We will first need a few lemmas.

The following result is standard. The proof given is intended for non-experts in the theory of algebraic groups and its first part is addressed to those with some knowledge of model theory.

Lemma 3.6. $\mathrm{SO}_{F}$ is connected and simple (as an abstract group), thus also $\mathbb{Q}_{p^{-}}$simple.

Proof. For the first assertion notice that $\mathrm{SO}_{F}$ over $\Omega$ is connected iff $\mathrm{SO}_{F}$ over $\mathbb{C}$ is connected (as $\Omega$ and $\mathbb{C}$ are elementarily equivalent) and by [35], page 35, 
3rd paragraph, $\mathrm{SO}_{F}$ over $\mathbb{C}$ is connected iff $\mathrm{SO}_{F}(\mathbb{C})$ is connected in the Hausdorff topology, which is a standard fact (see Gorbatsevich et al. [13], p. 23).

For the second assertion, we quote some results from Dieudonné 4 . Our group $\mathrm{SO}_{F}$ is $O_{7}^{+}(\Omega, F)$ in Dieudonné's notation. Again in his notation, $F$ has index $\nu \geq 1$ (see Section 9, page 17), since there is some non-zero $\left(x_{1}, \cdots, x_{7}\right) \in \Omega^{7}$ with $x_{1}^{2}+\cdots x_{7}^{2}=0$. So by Prop. 12 , page $28, O_{7}^{+}(\Omega, F)=C_{7}(\Omega, F)$, since every element of $\Omega$ is a square, where $C_{7}(\Omega, F)$ is the commutator group of $O_{7}^{+}(\Omega, F)$ (see Section 12, pp. 23-24). Then by Section 14, Th. 2, page 29, $P C_{7}(\Omega, F)$ is simple, where $\operatorname{PC}_{7}(\Omega, F)$ is the quotient of $C_{7}(\Omega, F)$ by the center of $O_{7}^{+}(\Omega, F)$ (see section 13, page 25). But this center is trivial (again see Section 13), so $\mathrm{SO}_{F}=O_{7}^{+}(\Omega, F)=C_{7}(\Omega, F)=P C_{7}(\Omega, F)$ is simple.

Lemma 3.7. $\mathbb{Q}_{p}-\operatorname{rank}\left(\mathrm{SO}_{F}\right) \geq 2$.

Proof. According to the definition in 35, page 85, the $\mathbb{Q}_{p}$-rank of $\mathrm{SO}_{F}$ is the maximal dimension of a $\mathbb{Q}_{p}$-subgroup of $\mathrm{SO}_{F}$, which can be diagonalized over $\mathbb{Q}_{p}$. It will then be enough to find a $\mathbb{Q}_{p}$-subgroup $H$ of the diagonal matrices in $\mathrm{GL}_{7}$ and a matrix $M$ in $\mathrm{GL}_{7}\left(\mathbb{Q}_{p}\right)$, such that $H$ has dimension $\geq 2$ and $M H M^{-1} \subseteq \mathrm{SO}_{F}$.

This follows from some standard facts on quadratic forms over $\mathbb{Q}_{p}$. We use Serre 31], Ch. IV, as a reference. By Serre [31, Theorem 6 in page 36 and Definitions 7, $4^{\prime}$ and Proposition $3^{\prime}$ in pp. 32-33, the form $F=x_{1}^{2}+\cdots+x_{7}^{2}$ over $\mathbb{Q}_{p}$ is equivalent to $F^{\prime}=x_{1} x_{2}+x_{3} x_{4}+g\left(x_{5}, g_{6}, x_{7}\right)$ over $\mathbb{Q}_{p}$ for some form $g$. Then if the matrix $M \in \mathrm{GL}_{7}\left(\mathbb{Q}_{p}\right)$ implements the equivalence and $\mathrm{SO}_{F^{\prime}}$ is the $\mathbb{Q}$-group of matrices in $\mathrm{GL}_{7}$ preserving $F^{\prime}$, then $M\left[\mathrm{SO}_{F^{\prime}}\left(\mathbb{Q}_{p}\right)\right] M^{-1} \subseteq \mathrm{SO}_{F}\left(\mathbb{Q}_{p}\right)$. Let $H$ be the group of all diagonal $7 \times 7$ matrices $\left(\lambda_{1}, \cdots, \lambda_{7}\right)$ such that $\lambda_{1} \lambda_{2}=1, \lambda_{3} \lambda_{4}=1$ and $\lambda_{5}=\lambda_{6}=\lambda_{7}=1$. Then $H$ is a $\mathbb{Q}$-group, thus a $\mathbb{Q}_{p}$-group, and has dimension 2 . Moreover, $H\left(\mathbb{Q}_{p}\right) \subseteq \mathrm{SO}_{F^{\prime}}\left(\mathbb{Q}_{p}\right)$, so $M\left[H\left(\mathbb{Q}_{p}\right)\right] M^{-1} \subseteq \mathrm{SO}_{F}\left(\mathbb{Q}_{p}\right)$ and thus, by Zariski density of $H\left(\mathbb{Q}_{p}\right)$ in $H$, we see that $M H M^{-1} \subseteq \mathrm{SO}_{F}$.

Note also that $\mathrm{SO}_{F}$ is semisimple, i.e., the (solvable) radical of $\mathrm{SO}_{F}$, which is defined to be the maximal normal connected solvable subgroup of $\mathrm{SO}_{F}$, is trivial, since $\mathrm{SO}_{F}$ is simple.

To be in a position to apply 3.3 , we now consider the algebraic universal cover of $\mathrm{SO}_{F}$, say $G$ (see the paragraph following 3.1.11 in Zimmer [35] or Margulis [26], 1.4, particularly 1.4.12). This maneuver is necessary to ensure the hypothesis that the group is algebraically simply connected.

Then $G$ is a connected semisimple $\mathbb{Q}_{p}$-group, which is also algebraically simply connected and almost $\mathbb{Q}_{p^{-}}$-simple. (Indeed, assume $N$ is a proper normal $\mathbb{Q}_{p^{-}}$ subgroup of $G$, and $\pi: G \rightarrow \mathrm{SO}_{F}$ is a $\mathbb{Q}_{p}$-isogeny, i.e., a $\mathbb{Q}_{p}$-epimorphism with finite kernel. Since $\mathrm{SO}_{F}$ is simple as an abstract group, either $\pi(N)=1$ or $\pi(N)=$ $\mathrm{SO}_{F}$. In the former case, $N$ is a finite subgroup of $G$. In the latter case, $N$ has finite index in $G$, which contradicts the fact that $G$ is connected.)

Moreover, by 3.7 and Margulis [26, 1.4.6(a), $\mathbb{Q}_{p}$-rank $(G) \geq 2$ (in applying 1.4.6(a) recall that semisimple $\Rightarrow$ reductive).

Let $\pi: G \rightarrow \mathrm{SO}_{F}$ as above be a $\mathbb{Q}_{p}$-isogeny. Then we can use $\pi$ to lift the action of $\mathrm{SO}_{F}\left(\mathbb{Q}_{p}\right)$ on $X$ to an action of $G\left(\mathbb{Q}_{p}\right)$ on $X$ defined by $g \cdot x=\pi(g) \cdot x$ (note that $\left.\pi\left(G\left(\mathbb{Q}_{p}\right)\right) \subseteq \mathrm{SO}_{F}\left(\mathbb{Q}_{p}\right)\right)$. We can also lift the cocycle $\alpha: \mathrm{SO}_{F}\left(\mathbb{Q}_{p}\right) \times X \rightarrow \operatorname{SO}_{F}\left(\mathbb{Q}_{q}\right)$ to the cocycle $\alpha_{1}: G\left(\mathbb{Q}_{p}\right) \times X \rightarrow \mathrm{SO}_{F}\left(\mathbb{Q}_{q}\right)$ given by $\alpha_{1}(g, x)=\alpha(\pi(g), x)$.

We claim that it suffices to show that $\alpha_{1}$ is equivalent to a cocycle taking values in a compact subgroup, say $K_{1}$, of $\mathrm{SO}_{F}\left(\mathbb{Q}_{q}\right)$. To see this argue as follows: In 
general, by Zimmer 35, page 34, paragraph 6 , when $H_{1}, H_{2}$ are $k$-groups and $\varphi: H_{1} \rightarrow H_{2}$ a $k$-morphism, then $\varphi\left(H_{1}\right)$ is a $k$-group and $\varphi\left(H_{1}(k)\right)$ is a subgroup of finite index in $\left(\varphi\left(H_{1}\right)\right)(k)$. Thus $\pi\left(G\left(\mathbb{Q}_{p}\right)\right)$ has finite index in $\mathrm{SO}_{F}\left(\mathbb{Q}_{p}\right)$ (actually in this particular case it turns out that $\pi\left(G\left(\mathbb{Q}_{p}\right)\right)=\mathrm{SO}_{F}\left(\mathbb{Q}_{p}\right)$ - see Margulis [26], page 42 , line 7 ). So by $2.7, \alpha$ is equivalent to a cocycle taking values in a compact subgroup of $\mathrm{SO}_{F}\left(\mathbb{Q}_{p}\right)$.

Now $G$ satisfies all the hypotheses of 3.3, but unfortunately we cannot take $H=\mathrm{SO}_{F}\left(\mathbb{Q}_{q}\right)$ and $\alpha=\alpha_{1}$ in 3.3. One problem is that $\alpha_{1}$ might be equivalent to a cocycle with range contained in a proper subgroup of the form $L\left(\mathbb{Q}_{q}\right)$ for a $\mathbb{Q}_{q}$-subgroup $L \subseteq H$. So we proceed as follows:

Call a cocycle $\beta: G\left(\mathbb{Q}_{p}\right) \times Y \rightarrow \mathrm{SO}_{F}\left(\mathbb{Q}_{q}\right)$ close to $\alpha: G\left(\mathbb{Q}_{p}\right) \times X \rightarrow \mathrm{SO}_{F}\left(\mathbb{Q}_{q}\right)$ if $Y$ is a finite extension of $X$ and if $\beta$ is equivalent to the lift of $\alpha$ to $Y$. (Note that this notion is not symmetric.)

Now let $\mathcal{C}$ denote the collection of all $\mathbb{Q}_{q}$-subgroups $L$ of $\mathrm{SO}_{F}$ such that there is a cocycle $\beta: G\left(\mathbb{Q}_{p}\right) \times Y \rightarrow \operatorname{SO}_{F}\left(\mathbb{Q}_{q}\right), \beta$ close to $\alpha_{1}$, with $\beta\left(G\left(\mathbb{Q}_{p}\right) \times Y\right) \subseteq L\left(\mathbb{Q}_{q}\right)$.

By the descending chain condition for algebraic groups, there is a minimal element $H$ in $\mathcal{C}$. Then choose a cocycle $\beta: G\left(\mathbb{Q}_{p}\right) \times Y \rightarrow \mathrm{SO}_{F}\left(\mathbb{Q}_{q}\right)$ which is close to $\alpha_{1}$ such that $\beta\left(G\left(\mathbb{Q}_{p}\right) \times Y\right) \subseteq H\left(\mathbb{Q}_{q}\right)$. By 2.6 , in order to show that $\alpha_{1}$ is equivalent to a cocycle taking values in a compact subgroup of $\mathrm{SO}_{F}\left(\mathbb{Q}_{q}\right)$, it is enough to show that the same holds for $\beta$.

First we will use the minimality of $H$ to deduce that $H$ must be connected, i.e, that $H=H^{0}$, where $H^{0}$ is the Zariski connected component of the identity of $H$. Now $H^{0}$ is a normal $\mathbb{Q}_{q}$-subgroup of $H$ of finite index, so $H^{0}\left(\mathbb{Q}_{q}\right)$ is a closed normal subgroup of finite index in $H\left(\mathbb{Q}_{q}\right)$. Then, by 2.5 , there exists a finite extension $Z$ of $Y$ such that if $\hat{\beta}$ is the lift of $\beta$ to $Z$, then there exists a cocycle $\gamma \sim \hat{\beta}$ such that $\gamma$ takes values in $H^{0}\left(\mathbb{Q}_{q}\right)$. But $\gamma$ is close to $\alpha_{1}$; and so, if $H^{0}$ is a proper subgroup of $H$, then this violates the minimality of $H$.

Now $H$ might not be semisimple, so let $R$ be the (solvable) radical of $H$ and $S=H / R, \tau: H \rightarrow S$ the canonical epimorphism. Then $S$ is a connected semisimple $\mathbb{Q}_{q}$-group and $\tau$ is a $\mathbb{Q}_{q}$-morphism. By Margulis [26], first two paragraphs of 0.24 in pages 20,21 , there are normal connected almost $\mathbb{Q}_{q}$-simple, $\mathbb{Q}_{q}$-subgroups of $S$, say $G_{1}, \cdots, G_{k}$, so that the multiplication operation from $G_{1} \times \cdots \times G_{k}$ into $S$ is an isogeny (i.e., an epimorphism with finite kernel), say $\rho$. Let $Z_{i}$ be the center of $G_{i}$ and let $S_{i}=G_{i} / Z_{i}$. Then since $G_{i}$ is connected and almost $\mathbb{Q}_{q}$-simple, every normal $\mathbb{Q}_{q}$-subgroup is finite, so contained in the center (see Margulis [26], 0.18), so $S_{i}$ is a connected $\mathbb{Q}_{q}$-simple, $\mathbb{Q}_{q}$-group, and since the kernel of $\rho$ is finite it must be contained in the center of $G_{1} \times \cdots \times G_{k}$, since this group is connected. So the kernel of $\rho$ is contained in the product $Z_{1} \times \cdots \times Z_{k}$ and there is a canonical $\mathbb{Q}_{p}$-epimorphism $\tau_{*}: S \rightarrow S_{1} \times \cdots \times S_{k}$. Denote by $\tau_{i}$ the composition of $\tau_{*}$ with the projection of $S_{1} \times \cdots \times S_{k}$ onto $S_{i}$.

Let $\sigma_{i}=\tau_{i} \circ \tau$ and $\beta_{i}=\sigma_{i} \circ \beta: G\left(\mathbb{Q}_{p}\right) \times Y \rightarrow S_{i}\left(\mathbb{Q}_{q}\right)$. Now all the hypotheses of 3.3 are clearly satisfied for $H=S_{i}, \alpha=\beta_{i}$. Recall that $\sigma_{i}\left(H\left(\mathbb{Q}_{q}\right)\right)$ has finite index in $S_{i}\left(\mathbb{Q}_{q}\right)$, as $\sigma_{i}$ maps $H$ onto $S_{i}$. View $\beta_{i}: G\left(\mathbb{Q}_{p}\right) \times Y \rightarrow \sigma_{i}\left(H\left(\mathbb{Q}_{q}\right)\right)$ as a cocycle into $\sigma_{i}\left(H\left(\mathbb{Q}_{q}\right)\right)$. Suppose that, viewed as a cocycle into $S_{i}\left(\mathbb{Q}_{q}\right), \beta_{i}$ is equivalent to a cocycle taking values in $L\left(\mathbb{Q}_{q}\right)$, where $L$ is a proper $\mathbb{Q}_{q}$-subgroup of $S_{i}$. Apply 2.4 to get that, as a cocycle into $\sigma_{i}\left(H\left(\mathbb{Q}_{q}\right)\right), \beta_{i}$ is equivalent to a cocycle with values in $L_{i}=\sigma_{i}\left(H\left(\mathbb{Q}_{q}\right)\right) \cap h L\left(\mathbb{Q}_{q}\right) h^{-1}$, for some $h \in S_{i}\left(\mathbb{Q}_{q}\right)$. But then, by 2.1 , there is a $\beta_{i}$-invariant Borel function $f: X \rightarrow \sigma_{i}\left(H\left(\mathbb{Q}_{q}\right)\right) / L_{i}$, so clearly there 
is a $\beta$-invariant Borel function $f^{\prime}: X \rightarrow H\left(\mathbb{Q}_{q}\right) / \sigma_{i}^{-1}\left(L_{i}\right)$, and so $\beta$ is equivalent to a cocycle taking values in $\sigma_{i}^{-1}\left(L_{i}\right)=\sigma_{i}^{-1}\left(h L\left(\mathbb{Q}_{q}\right) h^{-1}\right)$. But $L^{\prime}=h L h^{-1}$ is a proper $k$-subgroup of $S_{i}$, and so $\sigma_{i}^{-1}\left(L^{\prime}\right)$ is a proper $k$-subgroup of $H$, while $\sigma_{i}^{-1}\left(h L\left(\mathbb{Q}_{p}\right) h^{-1}\right)=\sigma_{i}^{-1}\left(L^{\prime}\left(\mathbb{Q}_{p}\right)\right)=\sigma_{i}^{-1}\left(L^{\prime}\right)\left(\mathbb{Q}_{p}\right)$, contradicting the minimality of $H$.

We conclude, for all $i$, that $\beta_{i}$ is equivalent to a cocycle with values in a compact subgroup $K_{i}$ of $S_{i}\left(\mathbb{Q}_{p}\right)$. Thus $\tau_{*} \circ \tau \circ \beta$ is equivalent to a cocycle taking values in $K_{1} \times \cdots \times K_{n}$. Then, as in the preceding argument, an application of 2.4 shows that $\beta$ is equivalent to cocycle $\gamma$ taking values into $A=\tau^{-1}\left(\tau_{*}^{-1}(K)\right)$, where $K$ is a compact subgroup of $S_{1}\left(\mathbb{Q}_{q}\right) \times \cdots \times S_{n}\left(\mathbb{Q}_{q}\right)$. In fact, $K$ is a conjugate of $K_{1} \times \cdots \times K_{n}$. Now $\tau_{*}^{-1}(K)$ is an extension of a compact group by a finite group, so it is amenable and $\tau^{-1}\left(\tau_{*}^{-1}(K)\right)$ is an extension of an amenable group by a solvable group (i.e., $R$ ), so $A$ is amenable (see [35], 4.6 and 4.1.2).

By Margulis [26, III 5.3 in page $130, G\left(\mathbb{Q}_{p}\right)$ is Kazhdan. So, by 3.1, the cocycle $\gamma$ is equivalent to a cocycle taking values in a compact subgroup of $A$, and so $\beta$ is equivalent to a cocycle taking values in a compact subgroup of $H\left(\mathbb{Q}_{q}\right)$ and the proof is complete.

The following cocycle reduction result will be needed in Section 6 .

Theorem 3.8. Let $n \geq 3$ and let $X$ be a standard Borel $\mathrm{SL}_{n}(\mathbb{R})$-space with an invariant, ergodic probability Borel measure. Let $m<n$ and let $H^{m}=\mathrm{SL}_{m}^{ \pm 1} /\left\{I_{m},-I_{m}\right\}$ be the group of all $m \times m$ matrices with determinant \pm 1 , modulo the subgroup $\left\{I_{m},-I_{m}\right\}$, where $I_{m}=$ the identity $m \times m$ matrix. Let $p$ be a prime or $\infty$ and let $\mathbb{Q}_{\infty}=\mathbb{R}$. If $\alpha: \mathrm{SL}_{n}(\mathbb{R}) \times X \rightarrow H^{m}\left(\mathbb{Q}_{p}\right)$ is a cocycle, then $\alpha$ is equivalent to a cocycle taking values in a compact subgroup of $H^{m}\left(\mathbb{Q}_{p}\right)$.

Proof. The argument is similar to that of 3.5, using 3.4 instead of 3.3, so we will only indicate the changes.

First since $\mathrm{SL}_{n}(\mathbb{C})$ is connected (see, e.g., Gorbatsevich et al. 13, page 22, Prop. 4.4), so is $\mathrm{SL}_{n}$. Also $\mathrm{SL}_{n}(\mathbb{R})$ is connected (same reference). Every normal subgroup of $\mathrm{SL}_{n}(K), K$ any field of characteristic 0 , is contained in its center which is finite (see Rotman [29], 8.9 and 9.4.6). In particular, $\mathrm{SL}_{n}$ is semisimple. Finally, $\mathbb{R}$-rank $\left(\mathrm{SL}_{n}(\mathbb{R})\right)=n-1$ (see Zimmer [35, page 85, first paragraph), so since $n \geq 3$ all the hypotheses about $G=\mathrm{SL}_{n}(\mathbb{R})$ in 3.4 are satisfied.

Then, by repeating the argument in 3.5 , we end up with a cocycle of the action of $\mathrm{SL}_{n}(\mathbb{R})$ on $X$ into a group of the form $S_{i}(k)$, where $k=\mathbb{Q}_{p}$ or $\mathbb{R}$, and $S_{i}$ is a connected $k$-simple, $k$-group for which there is a $k$-epimorphism from $H$ onto $S_{i}$, where $H$ is a connected $k$-subgroup of $H^{m}$. We have to conclude that this cocycle is equivalent to one with values in some compact subgroup of $S_{i}(k)$. Applying 3.4, this is the case unless $k=\mathbb{R}$ and there is a non-trivial $\mathbb{R}$-morphism $\rho$ from $\mathrm{SL}_{n}$ into $S_{i}$. But this is impossible, since the dimension of $S_{i}$ is less than or equal to that of $H^{m}$, which is $m^{2}-1$, and thus strictly smaller to that of $\mathrm{SL}_{n}$, which is $n^{2}-1$, and the kernel of $\rho$ is finite (see Humphreys [17], 7.4).

Finally, we need that $\mathrm{SL}_{n}(\mathbb{R})$, for $n \geq 3$, is Kazhdan (see de la Harpe and Valette [3], 2.a.4, page 21).

We conclude this section with the proof of a well-known fact that we will need in the next section. 
Proposition 3.9. Let $\mathbb{Z}\left[\frac{1}{p}\right]$ be the ring of rationals whose denominators (in reduced form) are powers of $p$. Then $\mathrm{SO}_{F}\left(\mathbb{Z}\left[\frac{1}{p}\right]\right)$ is a Kazhdan group.

Proof. We will need the following standard result:

Lemma 3.10. Let $G, H$ be lcsc groups, $\Gamma \subseteq G$ a lattice in $G$ and $f: G \rightarrow H$ a continuous epimorphism with compact kernel. Then $f(\Gamma)$ is a lattice in $H$.

Proof. Let $K=$ kernel of $f$. Then $K \Gamma=\Gamma K$ is a closed subgroup of $G$ and let $\phi$ be the canonical map from $G / \Gamma$ onto $G / K \Gamma$ given by $\varphi(h \Gamma)=h K \Gamma$. Then for the canonical actions of $G$ on $G / \Gamma$ and $G / K \Gamma$ we clearly have $\varphi(g \cdot x)=g \cdot \varphi(x)$, so if $\mu$ is a $G$-invariant measure on $G / \Gamma$, then $\nu=\varphi_{*}(\mu)$ is a $G$-invariant probability Borel measure on $G / K \Gamma$. Now let $\psi: G / K \Gamma \rightarrow H / f(\Gamma)$ be the canonical bijection given by

$$
\psi(h K \Gamma)=f(h) f(\Gamma) .
$$

Then, as $\psi(g \cdot x)=f(g) \cdot \psi(x)$, it follows that $\psi_{*}(\nu)$ is $H$-invariant. Finally it is easy to check that $f(\Gamma)$ is discrete in $H$.

Now take $\Gamma=\mathrm{SO}_{F}\left(\mathbb{Z}\left[\frac{1}{p}\right]\right)$ and let $K=\mathrm{SO}_{F}(\mathbb{R}), H=\mathrm{SO}_{F}\left(\mathbb{Q}_{p}\right)$. Then $K$ is compact. Define $\delta: \Gamma \rightarrow K \times H$ by $\delta(x)=(x, x)$. Then by Zimmer 35], 10.1.1 (with $G=\mathrm{SO}_{F}, S=\{p\}$ in his notation), $\delta(\Gamma)$ is a lattice in $K \times H$, so if $\rho: K \times H \rightarrow H$ is the projection, by the previous lemma, $\rho(\delta(\Gamma))=\Gamma$ is a lattice in $\operatorname{SO}_{F}\left(\mathbb{Q}_{p}\right)$. By Margulis [26], III 5.3 in page $130, \mathrm{SO}_{F}\left(\mathbb{Q}_{p}\right)$ is a Kazhdan group and by Zimmer [35], 7.4.3, every lattice in a Kazhdan group is also a Kazhdan group, so $\mathrm{SO}_{F}\left(\mathbb{Z}\left[\frac{1}{p}\right]\right)$ is a Kazhdan group.

\section{EMBEDding Borel SETS UNDER INCLUSION}

Our goal in this section is to prove the first main theorem of this paper.

Theorem 4.1. The partial ordering of Borel sets under inclusion can be embedded in the partial (pre)order of countable Borel equivalence relations under Borel reducibility. More precisely, there is a map $A \mapsto E_{A}$ assigning to each Borel subset of $2^{\mathbb{N}}$, A, a countable Borel equivalence relation, $E_{A}$, so that

$$
A_{1} \subseteq A_{2} \Leftrightarrow E_{A_{1}} \leq{ }_{B} E_{A_{2}} .
$$

The proof of 4.1 is based on the following result.

Theorem 4.2. There is a map $x \mapsto E_{x}$ assigning to each $x \in 2^{\mathbb{N}}$ a countable Borel equivalence relation $E_{x}$ on $2^{\mathbb{N}}$ such that:

(i) $E_{x}$ admits an ergodic, non-atomic, invariant measure $\mu_{x}$.

(ii) If $x_{0} \neq x_{1}$ and $f: 2^{\mathbb{N}} \rightarrow 2^{\mathbb{N}}$ is a Borel function such that $y E_{x_{0}} z \Rightarrow$ $f(y) E_{x_{1}} f(z)$, then there is a Borel $E_{x_{0}}$-invariant set $M$ of $\mu_{x_{0}}$-measure 1 such that $f(M)$ is contained in a single $E_{x_{1}}$-class. In particular, for any Borel set $N$ of $\mu_{x_{0}}$-measure $1, E_{x_{0}} \mid N \not \mathbb{Z}_{B} E_{x_{1}}$.

(iii) The assignment $x \mapsto E_{x}$ is "Borel", in the sense that $E \subseteq\left(2^{\mathbb{N}}\right)^{3}$ below is Borel:

$$
(x, y, z) \in E \Leftrightarrow y E_{x} z .
$$

Moreover $x \mapsto \mu_{x}$ is Borel. 
Granting 4.2 we can prove 4.1 as follows:

To each Borel set $A \subseteq 2^{\mathbb{N}}$ assign the following equivalence relation:

$$
E_{A}=\bigsqcup_{x \in A} E_{x}
$$

that is, the direct sum of the $E_{x}$ with $x \in A$. This is precisely defined as follows: $E_{A}$ is an equivalence relation on $A \times 2^{\mathbb{N}}$, and

$$
(x, y) E_{A}\left(x^{\prime}, z\right) \Leftrightarrow x=x^{\prime} \& y E_{x} z .
$$

Then $E_{A}$ is Borel and, since there is a Borel bijection of $A \times 2^{\mathbb{N}}$ with $2^{\mathbb{N}}$, we may, if we wish, think of $E_{A}$ as being an equivalence relation on $2^{\mathbb{N}}$. It is clear that if $A_{1} \subseteq A_{2}$, then $E_{A_{1}} \leq_{B} E_{A_{2}}$ via the identity function. Now assume that $A_{1} \nsubseteq A_{2}$ and let $x \in A_{1} \backslash A_{2}$. Then clearly $E_{x} \leq_{B} E_{A_{1}}$ (via $y \mapsto(x, y)$ ). On the other hand, we claim that $E_{x} \mathbb{Z}_{B} E_{A_{2}}$, so $E_{A_{1}} \mathbb{Z}_{B} E_{A_{2}}$. Because, otherwise, there is a Borel function $f: 2^{\mathbb{N}} \rightarrow A_{2} \times 2^{\mathbb{N}}$ with $y E_{x} z \Leftrightarrow f(y) E_{A_{2}} f(z)$, and so, in particular, $y E_{x} z \Rightarrow \pi(f(y))=\pi(f(z))$, where $\pi(a, b)=a$. By ergodicity, there is a Borel set $M$ of $\mu_{x}$-measure 1 with $\pi(f(y))$ constant for $y \in M$, say with value $\pi(f(y))=x_{0}$. Then $y \mapsto \rho(f(y))$, where $\rho(a, b)=b$, is a Borel reduction of $E_{x} \mid M$ into $E_{x_{0}}$, which contradicts 4.2(ii), as $x \neq x_{0}$.

We will devote the rest of this section to the proof of Theorem 4.2.

For any infinite countable group $\Gamma$, consider the compact Polish space $2^{\Gamma}$ of all functions from $\Gamma$ into $2=\{0,1\}$ with the product topology (so that it is homeomorphic to $\left.2^{\mathbb{N}}\right)$. $\Gamma$ acts on $2^{\Gamma}$ by (left) shift: $g \cdot x(h)=x\left(g^{-1} h\right)$. We denote by $E(\Gamma, 2)$ the associated equivalence relation

$$
x E(\Gamma, 2) y \Leftrightarrow \exists g(g \cdot x=y) .
$$

We also let $F(\Gamma, 2)$ be the restriction of $E(\Gamma, 2)$ to the free part of the action, i.e.,

$$
F(\Gamma, 2)=E(\Gamma, 2) \mid \operatorname{Fr}_{\Gamma}
$$

where $\operatorname{Fr}_{\Gamma}=\left\{x \in 2^{\Gamma}: \forall g \neq 1(g \cdot x \neq x)\right\}$. We also denote by $\mu_{\Gamma}$ the product measure on $2^{\Gamma}$ (where each bit in $\{0,1\}$ has measure $1 / 2$ ). It is clearly invariant under the shift action and it is non-atomic. The next lemma is quite standard.

Lemma 4.3. $\mu_{\Gamma}\left(\operatorname{Fr}_{\Gamma}\right)=1$.

Proof. It is clearly enough to show that for each $g \neq 1, X_{g}=\left\{x \in 2^{\Gamma}: \forall h(x(g h)=\right.$ $x(h))\}$ has $\mu_{\Gamma}$-measure 0 . It is clear that if $x \in X_{g}$, then $x$ is constant on each right coset $\langle g\rangle h, h \in \Gamma$, of the subgroup $\langle g\rangle$ generated by $g$. Since either $\langle g\rangle$ is infinite or there are infinitely many cosets, this implies that $\mu_{\Gamma}\left(X_{g}\right)=0$.

If $\Delta$ is a subgroup of $\Gamma$, then also $\Delta$ acts on $2^{\Gamma}$ by left shift and we denote by $E(\Delta, \Gamma, 2)$ the corresponding equivalence relation, so that

$$
x E(\Delta, \Gamma, 2) y \Leftrightarrow \exists g \in \Delta(g \cdot x=y) .
$$

The following lemma is also standard. In fact, the action of $\Gamma$ on $2^{\Gamma}$ turns out to be mixing, a property which is inherited by infinite subgroups of $\Gamma$, and which implies ergodicity. For the sake of completeness, we include a proof which does not depend on understanding the mixing property. 
Lemma 4.4. For any infinite subgroup $\Delta$ of $\Gamma, \mu_{\Gamma}$ is $E(\Delta, \Gamma, 2)$-ergodic.

Proof. Suppose $X \subseteq 2^{\Gamma}$ is Borel and $E(\Delta, \Gamma, 2)$-invariant. Enumerate $\Gamma=$ $\left\{\gamma_{0}, \gamma_{1}, \cdots, \gamma_{n}, \cdots\right\}$. Then for each $n$, choose $\delta_{n} \in \Delta$ such that $\delta_{n}$ is not one of $\gamma_{i} \gamma_{j}^{-1}$, for $i, j<n$. Then $\delta_{n}\left\{\gamma_{0}, \cdots, \gamma_{n-1}\right\} \cap\left\{\gamma_{0}, \cdots, \gamma_{n-1}\right\}=\emptyset$. Consider the Boolean algebra $\mathcal{A}$ of clopen subsets of $2^{\Gamma}$. Denote by MALG $\mu_{\mu_{\Gamma}}$ the measure algebra of $\mu_{\Gamma}$, i.e., the Boolean algebra of Borel subsets of $2^{\Gamma}$ modulo sets of $\mu_{\Gamma}$-measure 0 . Then $\mathcal{A}$ is dense in MALG $\mu_{\mu_{\Gamma}}$ (see, e.g., Kechris [21], 17.43]), so for each $n$ there is $A_{n} \in \mathcal{A}$ with $\mu_{\Gamma}\left(X+A_{n}\right) \rightarrow 0$, where + denotes here the symmetric difference of two sets. Now each $A_{n}$ is a finite union of sets of the form

$$
N_{s}=\left\{x \in 2^{\Gamma}: x \mid F=s\right\},
$$

where $s: F \rightarrow 2$, for some finite subset $F \subseteq \Gamma$. Call $F$ the support of $N_{s}$ and if $A_{n}=$ $N_{s_{1}} \cup \cdots \cup N_{s_{k}}$, with the support of $N_{s_{i}}$ equal to $F_{i}$, call $F_{1} \cup \cdots \cup F_{k}$ the support of $A_{n}$. Say it is included in $\left\{\gamma_{0}, \cdots, \gamma_{k_{n}-1}\right\}$. Then it is clear that if $\epsilon_{n}=\delta_{k_{n}}^{-1}, A_{n}$ and $\epsilon_{n} \cdot A_{n}$ are independent for $\mu_{\Gamma}$, so $\mu_{\Gamma}\left(A_{n} \cap \epsilon_{n} \cdot A_{n}\right)=\mu_{\Gamma}\left(A_{n}\right) \mu_{\Gamma}\left(\epsilon_{n} \cdot A_{n}\right)=\mu_{\Gamma}\left(A_{n}\right)^{2}$ by the invariance of $\mu_{\Gamma}$. Also $\mu_{\Gamma}\left(A_{n}+X\right)=\mu_{\Gamma}\left(\epsilon_{n} \cdot\left(A_{n}+X\right)\right)=\mu_{\Gamma}\left(\epsilon_{n} \cdot A_{n}+X\right)$, as $\epsilon_{n} \cdot X=X$. So $A_{n} \rightarrow X$ and $\epsilon_{n} \cdot A_{n} \rightarrow X$ in the measure algebra, so, as intersection is continuous (see Kechris [21], 17.43), $A_{n} \cap \epsilon_{n} \cdot A_{n} \rightarrow X$ and thus $\mu_{\Gamma}\left(A_{n} \cap \epsilon_{n} \cdot A_{n}\right)=$ $\mu_{\Gamma}\left(A_{n}\right)^{2} \rightarrow \mu(X)$. But also $\mu_{\Gamma}\left(A_{n}\right) \rightarrow \mu_{\Gamma}(X)$, so $\mu_{\Gamma}(X)^{2}=\mu_{\Gamma}(X)$, i.e., $\mu_{\Gamma}(X)=0$ or 1 .

Recall that $\mathrm{SO}_{F}$ is the $\mathbb{Q}$-group of all $7 \times 7$ matrices $A$ with $\operatorname{det}(A)=1, A A^{t}=I$. For each non-empty subset $S$ of primes, let $\mathbb{Z}\left[S^{-1}\right]$ be the ring of rationals whose denominators (in reduced form) have prime factors in $S$, and let

$$
\Gamma_{S}=\mathrm{SO}_{F}\left(\mathbb{Z}\left[S^{-1}\right]\right)
$$

be the set of all $7 \times 7$ matrices in $\mathrm{SO}_{F}$ with coefficients in $\mathbb{Z}\left[S^{-1}\right]$. Let $E_{S}=F\left(\Gamma_{S}, 2\right)$ and $X_{S}=\operatorname{Fr}_{\Gamma_{S}}, \mu_{S}=\mu_{\Gamma_{S}}$. Then we have the following lemma:

Lemma 4.5. If $S \nsubseteq \nsubseteq T$, and $f: X_{S} \rightarrow X_{T}$ is Borel such that $x E_{S} y \Rightarrow f(x) E_{T} f(y)$, then there is a Borel $E_{S}$-invariant set $M$ with $\mu_{S}(M)=1$ such that $f$ maps $M$ into a single $E_{T}$-class.

Granting this, we can prove 4.2 as follows: Let $\pi$ be a Borel function from $2^{\mathbb{N}}$ into the power set of the primes (which we identify with $2^{P}, P=$ the set of primes) so that each $\pi(x)$ is infinite and

$$
x \neq y \Rightarrow \pi(x) \cap \pi(y) \text { is finite, }
$$

i.e., $\{\pi(x)\}_{x \in 2^{\mathbb{N}}}$ is an almost disjoint family of subsets of primes. (The existence of such a Borel function $\pi$ is a standard fact and here is a quick proof: It is clearly enough to replace $P$ by $\mathbb{N}$. Fix a bijection \langle\rangle$: 2^{<\mathbb{N}} \rightarrow \mathbb{N}$ of the set of finite sequences $\left\langle s_{0}, \cdots, s_{n-1}\right\rangle$ from $\mathbb{N}$ with $\mathbb{N}$, and for $x \in 2^{\mathbb{N}}$ let $\pi(x)=\{\langle x(0), \cdots, x(n-1)\rangle: n \in$ $\mathbb{N}\} \subseteq \mathbb{N}$.) For $x \in 2^{\mathbb{N}}$, put

$$
E_{x}=E_{\pi(x)}
$$

and $\mu_{x}=\mu_{\pi(x)}$. Then (i)-(iii) of 4.2 are clear. (Literally $E_{x}$ is not an equivalence relation on $2^{\mathbb{N}}$ but it can be considered as such by fixing Borel bijections of each $X_{\pi(x)}$ with $2^{\mathbb{N}}$, uniformly in $x$.) 
Proof of Lemma 4.5. Fix $p \in S \backslash T$, and consider $\Gamma_{p}=\Gamma_{\{p\}} \subseteq \Gamma_{S}$, i.e., $\Gamma_{p}=$ $\mathrm{SO}_{F}\left(\mathbb{Z}\left[\frac{1}{p}\right]\right)$. By 4.4 the measure $\mu_{S}$ is ergodic and invariant for the action of $\Gamma_{p}$ on $X_{S}$. We can now define the following strict Borel cocycle $\alpha: \Gamma_{p} \times X_{S} \rightarrow \Gamma_{T}$ :

$$
\begin{gathered}
\alpha(g, x)=\text { the unique } h \in \Gamma_{T} \text { such that } \\
\\
h \cdot f(x)=f(g \cdot x)
\end{gathered}
$$

which exists since $x E_{S} y \Rightarrow f(x) E_{T} f(y)$, and the action of $\Gamma_{T}$ is free.

Since, by 3.9, $\Gamma_{p}$ is a Kazhdan group, it follows from 3.2 that $\alpha \sim \beta$, where $\beta\left(\Gamma_{p} \times X_{S}\right) \subseteq \Lambda_{T}$, with $\Lambda_{T} \subseteq \Gamma_{T}$ a finitely generated subgroup of $\Gamma_{T}$. So there is a finite $T_{0} \subseteq T$ such that $\Lambda_{T} \subseteq \Gamma_{T_{0}}$. Say $T_{0}=\left\{p_{1}, \cdots, p_{n}\right\}$. By Zimmer [35, B. 9 (on page 200), we can also assume that $\beta$ is a strict cocycle.

Now $\Gamma_{T_{0}}=\mathrm{SO}_{F}\left(\mathbb{Z}\left[T_{0}^{-1}\right]\right)$ is a discrete subgroup of $\mathrm{SO}_{F}(\mathbb{R}) \times \mathrm{SO}_{F}\left(\mathbb{Q}_{p_{1}}\right) \times \cdots \times$ $\mathrm{SO}_{F}\left(\mathbb{Q}_{p_{n}}\right)$ (as $\mathbb{Z}\left[T_{0}^{-1}\right]$ is a discrete subring of $\mathbb{R} \times \mathbb{Q}_{p_{1}} \times \cdots \times \mathbb{Q}_{p_{n}}$ ) and thus of $\mathrm{SO}_{F}\left(\mathbb{Q}_{p_{1}}\right) \times \cdots \times \mathrm{SO}_{F}\left(\mathbb{Q}_{p_{n}}\right)$, as $\mathrm{SO}_{F}(\mathbb{R})$ is compact. (Here an element $g \in \Gamma_{T_{0}}$ is identified with $(g, \cdots, g)$ in this product and similarly for elements of $\mathbb{Z}\left[T_{0}^{-1}\right]$.) Also $\Gamma_{p}=\mathrm{SO}_{F}\left(\mathbb{Z}\left[\frac{1}{p}\right]\right)$ is a lattice in $\mathrm{SO}_{F}(\mathbb{R}) \times \mathrm{SO}_{F}\left(\mathbb{Q}_{p}\right)$, by Zimmer 35], 10.1.1 (since $\mathrm{SO}_{F}$ is a connected semisimple $\mathbb{Q}$-group), and so by 3.10 , it is a lattice in $\mathrm{SO}_{F}\left(\mathbb{Q}_{p}\right)$.

Consider the action of $\Gamma_{p}$ on $\left(X_{S}, \mu_{S}\right)$ and the strict cocycle $\beta: \Gamma_{p} \times X_{S} \rightarrow \Gamma_{T_{0}}$. Using Section 2 (paragraph following 2.2), consider the induced Borel action of $\mathrm{SO}_{F}\left(\mathbb{Q}_{p}\right)$ on $Y$ (which also has an invariant, ergodic measure) and the induced strict cocycle $\hat{\beta}: \mathrm{SO}_{F}\left(\mathbb{Q}_{p}\right) \times Y \rightarrow \Gamma_{T_{0}}$. Viewing $\hat{\beta}$ as a cocycle $\hat{\beta}: \mathrm{SO}_{F}\left(\mathbb{Q}_{p}\right) \times$ $Y \rightarrow \mathrm{SO}_{F}\left(\mathbb{Q}_{p_{1}}\right) \times \cdots \times \mathrm{SO}_{F}\left(\mathbb{Q}_{p_{n}}\right)$ and letting $\pi_{i}$ be the projection of $\mathrm{SO}_{F}\left(\mathbb{Q}_{p_{1}}\right) \times$ $\cdots \times \mathrm{SO}_{F}\left(\mathbb{Q}_{p_{n}}\right)$ to $\mathrm{SO}_{F}\left(\mathbb{Q}_{p_{i}}\right), i=1, \cdots, n$, consider the cocycle $\hat{\beta}_{i}=\pi_{i} \circ \hat{\beta}$ : $\mathrm{SO}_{F}\left(\mathbb{Q}_{p}\right) \times Y \rightarrow \mathrm{SO}_{F}\left(\mathbb{Q}_{p_{i}}\right)$. By 3.5, $\hat{\beta}_{i} \sim \gamma_{i}$, where $\gamma_{i}$ is a Borel cocycle with $\gamma_{i}\left(\mathrm{SO}_{F}\left(\mathbb{Q}_{p}\right) \times Y\right) \subseteq K_{i}, K_{i}$ a compact subgroup of $\mathrm{SO}_{F}\left(\mathbb{Q}_{p_{i}}\right)$. Set $K=K_{1} \times$ $\cdots \times K_{n}$. Then $\hat{\beta} \sim \gamma$ where $\gamma: \mathrm{SO}_{F}\left(\mathbb{Q}_{p}\right) \times Y \rightarrow \mathrm{SO}_{F}\left(\mathbb{Q}_{p_{1}}\right) \times \cdots \times \mathrm{SO}_{F}\left(\mathbb{Q}_{p_{n}}\right)$ is a Borel cocycle with $\gamma\left(\operatorname{SO}_{F}\left(\mathbb{Q}_{p}\right) \times Y\right) \subseteq K$. Then by 2.4, now viewing $\hat{\beta}$ as a cocycle into $\Gamma_{T_{0}}$, we have that $\hat{\beta} \sim \beta^{\prime}$ where $\beta^{\prime}: \mathrm{SO}_{F}\left(\mathbb{Q}_{p}\right) \times Y \rightarrow \Gamma_{T_{0}}$ is a Borel cocycle with $\beta^{\prime}\left(\mathrm{SO}_{F}\left(\mathbb{Q}_{p}\right) \times Y\right) \subseteq \Delta_{0}, \Delta_{0}$ a finite subgroup of $\Gamma_{T_{0}}$. Now using 2.3 , this shows that $\beta \sim \beta_{0}$, where $\beta_{0}: \Gamma_{p} \times X_{S} \rightarrow \Gamma_{T_{0}}$ is a Borel cocycle with $\beta_{0}\left(\Gamma_{p} \times X_{S}\right) \subseteq \Delta_{0}$ and so $\alpha \sim \beta_{0}$. Thus there is a Borel function $A: X_{S} \rightarrow \Gamma_{T}$ such that for all $g \in \Gamma_{p}, \alpha(g, x)=A(g \cdot x) \beta_{0}(g, x) A(x)^{-1}, \mu_{S^{-}}$a.e. $(x)$. Since $\Gamma_{T}$ is countable, find some Borel set $N_{0} \subseteq X_{S}$ of positive $\mu_{S}$-measure, such that $A$ is constant, say with value $A_{0}$, on $N_{0}$, and, since $\Gamma_{p}$ is countable, we can assume that $\alpha(g, x)=A(g \cdot x) \beta_{0}(g, x) A(x)^{-1}$, for all $g \in \Gamma_{p}, x \in N_{0}$. Then if $x, g \cdot x \in N_{0}$ for some $g \in \Gamma_{p}, \alpha(g, x)=A_{0} \beta_{0}(g, x) A_{0}^{-1} \in A_{0} \Delta_{0} A_{0}^{-1}$. Since $\alpha(g, x) \cdot f(x)=f(g \cdot x)$ it follows that as $g$ varies over $\Gamma_{p}$ so that $g \cdot x \in N_{0}, f(g \cdot x)$ takes only finitely many values. Let $M_{0}=\Gamma_{p} \cdot N_{0}$ be the $\Gamma_{p}$-saturation of $N_{0}$. Then $M_{0}$ has $\mu_{S}$-measure 1 by ergodicity. Moreover, the map on $M_{0}$ defined by $F(x)=f\left(\left(\Gamma_{p} \cdot x\right) \cap N_{0}\right)$ is $\Gamma_{p}$-invariant and assigns in a Borel way to $x$ a finite subset of $X_{T}$, so, by ergodicity again, it is fixed on a Borel set $M_{1} \subseteq M_{0}$ of $\mu_{S}$-measure 1 . Note that if $x_{0} \in$ $M_{0}$, then $F(x)$ is a finite subset of the $E_{T}$-class which $f$ assigns to the $E_{S}$-class containing $\Gamma_{p} \cdot x$. Hence $f$ maps $M_{1}$ into a single $E_{T}$-class. Finally, let $M=\Gamma_{S} \cdot M_{1}$. Then clearly $M$ is a Borel $E_{S}$-invariant subset of $\mu_{S}$-measure 1 which is mapped into a single $E_{T}$-class. 


\section{The DESCRIPTIVE COMPLEXITY OF BoREL REDUCIBILITY}

Let us first fix a parametrization of Borel relations on $\mathbb{N}^{\mathbb{N}}$. This consists of a set $D \subseteq 2^{\mathbb{N}}$ and two sets $P, S \subseteq\left(\mathbb{N}^{\mathbb{N}}\right)^{3}$ such that

(i) $D$ is $\boldsymbol{\Pi}_{1}^{1}, P$ is $\boldsymbol{\Pi}_{1}^{1}$ and $S$ is $\boldsymbol{\Sigma}_{1}^{1}$.

(ii) For $d \in D, P_{d}=S_{d}$ (where $P_{d}=\{(x, y):(d, x, y) \in D\}$ ). Denote by $D_{d}$ the set $P_{d}\left(=S_{d}\right)$, for $d \in D$.

(iii) $\left\{D_{d}: d \in 2^{\mathbb{N}}\right\}=\left\{B \subseteq\left(\mathbb{N}^{\mathbb{N}}\right)^{2}: B\right.$ is Borel $\}$.

(iv) If $A \subseteq X \times\left(\mathbb{N}^{\mathbb{N}}\right)^{2}, X$ a Polish space, is Borel, there is a Borel function $p: X \rightarrow 2^{\mathbb{N}}$, such that $A_{x}=D_{p(x)}$, for all $x \in X$ (see Kechris [21], 35.5).

It is straightforward, using Kechris [21], 29.19, that

$$
\mathcal{E}=\left\{d \in D: D_{d} \text { is a countable equivalence relation }\right\}
$$

is $\Pi_{1}^{1}$ and it is also easy to check that

$$
\begin{aligned}
\mathcal{E}_{\leq} & =\left\{(d, e) \in D^{2}: d, e \in \mathcal{E} \& D_{d} \leq_{B} D_{e}\right\}, \\
\mathcal{E}_{\sim} & =\left\{(d, e) \in D^{2}: d, e \in \mathcal{E} \& D_{d} \sim_{B} D_{e}\right\}
\end{aligned}
$$

are $\boldsymbol{\Sigma}_{\mathbf{2}}^{\mathbf{1}}$.

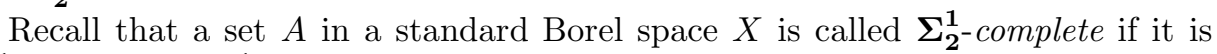
$\boldsymbol{\Sigma}_{\mathbf{2}}^{\mathbf{1}}$ and for any $\boldsymbol{\Sigma}_{\mathbf{2}}^{\mathbf{1}}$ set $B \subseteq Y, Y$ a standard Borel space, there is a Borel function $f: Y \rightarrow X$ such that $B=f^{-1}(A)$. The following therefore computes the exact complexity of $\mathcal{E}_{\leq}, \mathcal{E}_{\sim}$.

Theorem 5.1. The sets $\mathcal{E}_{\leq}, \mathcal{E}_{\sim}$ are $\boldsymbol{\Sigma}_{\mathbf{2}}^{1}$-complete. In other words, the notions of Borel reducibility and bi-reducibility of countable Borel equivalence relations are $\mathbf{\Sigma}_{\mathbf{2}}^{\mathbf{1} \text {-complete. }}$

Proof. We will prove in fact the stronger statement that there is a fixed countable Borel equivalence relation $E^{*}$ such that the countable Borel equivalence relations to which $E^{*}$ Borel reduces, resp. which are Borel bi-reducible with $E^{*}$, are both

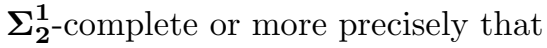

$$
\begin{aligned}
\mathcal{E}_{\leq}^{*} & =\left\{d \in D: d \in \mathcal{E} \& E^{*} \leq_{B} D_{d}\right\}, \\
\mathcal{E}_{\sim}^{*} & =\left\{d \in D: d \in \mathcal{E} \& E^{*} \sim_{B} D_{d}\right\}
\end{aligned}
$$

are $\boldsymbol{\Sigma}_{2}^{1}$-complete. The relation $E^{*}$ is simply defined as follows: For any countable Borel equivalence relation $E$ on $X$ let $c E$ be the sum of continuum many copies of $E$. We view $c E$ as defined on $\mathbb{N}^{\mathbb{N}} \times X$ by

$$
(a, x) c E(b, y) \Leftrightarrow a=b \& x E y .
$$

By trivially modifying 4.2 , fix a map $x \mapsto E_{x}$ from $\mathbb{N}^{\mathbb{N}}$ into Borel equivalence relations on $\mathbb{N}^{\mathbb{N}}$, satisfying (i)-(iii) of 4.2 (with $\mathbb{N}^{\mathbb{N}}$ replacing $2^{\mathbb{N}}$ ), and put

$$
E_{x}^{*}=c E_{x}
$$

and

$$
E^{*}=\bigsqcup_{x} E_{x}^{*}
$$

(where we can of course view all of these as living on $\mathbb{N}^{\mathbb{N}}$ ). 
Consider a tree $T$ on $\mathbb{N} \times \mathbb{N}$ (see Kechris 21, 2.1). Each such $T$ defines a closed subset $[T] \subseteq\left(\mathbb{N}^{\mathbb{N}}\right)^{2}$ given by

$$
[T]=\left\{(x, y) \in\left(\mathbb{N}^{\mathbb{N}}\right)^{2}: \forall n(x|n, y| n) \in T\right\} .
$$

Associate with $T$ the following countable Borel equivalence relation, where $\boldsymbol{\Delta}$ is the equality relation on $\mathbb{N}^{\mathbb{N}}$ :

$$
E_{T}=\bigsqcup_{(x, y) \in[T]} E_{x}^{*} \sqcup \bigsqcup_{(x, y) \notin[T]} \boldsymbol{\Delta} .
$$

Let us say that a closed set $F \subseteq\left(\mathbb{N}^{\mathbb{N}}\right)^{2}$ admits a full Borel uniformization if there is a Borel function $f: \mathbb{N}^{\mathbb{N}} \rightarrow \mathbb{N}^{\mathbb{N}}$ such that for every $x,(x, f(x)) \in F$. The main observation that we will use in the calculation of the complexity of $\mathcal{E}_{\leq}^{*}, \mathcal{E}_{\sim}^{*}$ is now the following:

Lemma 5.2. For each tree $T$ we have

$$
\begin{aligned}
E^{*} \leq_{B} E_{T} & \Leftrightarrow E^{*} \sim_{B} E_{T} \\
& \Leftrightarrow[T] \text { admits a full Borel uniformization. }
\end{aligned}
$$

Proof. Since $E_{T} \leq_{B} \bigsqcup_{(x, y) \in\left(\mathbb{N N}^{2}{ }^{2}\right.} E_{x}^{*} \leq_{B} \bigsqcup_{x}\left(c E_{x}^{*}\right) \cong_{B} \bigsqcup_{x} c\left(c E_{x}\right) \cong_{B} \bigsqcup_{x} c E_{x}=E^{*}$, where $\cong_{B}$ denotes Borel isomorphism, clearly

$$
E^{*} \leq_{B} E_{T} \Leftrightarrow E^{*} \sim_{B} E_{T} .
$$

Now assume that $[T]$ admits a full Borel uniformization, say $f$. View $E_{x}^{*}$ as being defined on $\mathbb{N}^{\mathbb{N}}$ and $E^{*}$ as defined on $\mathbb{N}^{\mathbb{N}} \times \mathbb{N}^{\mathbb{N}}$ by

$$
(x, y) E^{*}\left(x^{\prime}, z\right) \Leftrightarrow x=x^{\prime} \& y E_{x}^{*} z,
$$

and let $g: \mathbb{N}^{\mathbb{N}} \times \mathbb{N}^{\mathbb{N}} \rightarrow\left(\mathbb{N}^{\mathbb{N}} \times \mathbb{N}^{\mathbb{N}}\right) \times \mathbb{N}^{\mathbb{N}}$ be defined by

$$
g(x, y)=(x, f(x), y) .
$$

Then $g$ is a Borel reduction of $E^{*}$ into $E_{T}$.

Conversely, let the Borel function $g: \mathbb{N}^{\mathbb{N}} \times \mathbb{N}^{\mathbb{N}} \rightarrow\left(\mathbb{N}^{\mathbb{N}} \times \mathbb{N}^{\mathbb{N}}\right) \times \mathbb{N}^{\mathbb{N}}$ reduce $E^{*}$ into $E_{T}$. Then the function $g_{x}(y)=g(x, y)$ is a Borel reduction of $E_{x}^{*}$ into $E_{T}$. We clearly have a Borel function $\rho$ reducing each $E_{x}$ into $E_{x}^{*}(\rho$ is defined independently of $x$ ), so $g_{x} \circ \rho$ is a Borel reduction of $E_{x}$ into $E_{T}$, thus in particular

$$
y E_{x} z \Rightarrow \pi_{1}\left(\left(g_{x} \circ \rho\right)(y)\right)=\pi_{1}\left(\left(g_{x} \circ \rho\right)(z)\right)
$$

where $\pi_{1}(u, v, w)=(u, v)$. Since $\mu_{x}$ (as in 4.2(i)) is ergodic, this implies that there is a set $M_{x}$ of $\mu_{x}$-measure 1 , so that $\pi_{1} \circ g_{x} \circ \rho$ is constant on $M_{x}$, say with value $(u(x), f(x))$. But then, if $\pi_{2}(u, v, w)=w, \pi_{2} \circ g_{x} \circ \rho$ is a Borel reduction of $E_{x} \mid M_{x}$ into $E_{u(x)}^{*}$, if $(u(x), f(x)) \in[T]$, or into $\boldsymbol{\Delta}$, if $(u(x), f(x)) \notin[T]$. By applying ergodicity one more time, we conclude that $(u(x), f(x)) \in[T]$ and there is a Borel set of $\mu_{x}$-measure $1, N_{x}$, so that $E_{x} \mid N_{x} \leq_{B} E_{u(x)}$, so by $4.2(\mathrm{ii}), u(x)=x$. So clearly $f$ is a full uniformization of $[T]$ and it only remains to check that it is Borel. To see this, notice that the graph of $f$ is given by

$$
\begin{aligned}
(x, y) \in G_{f} & \Leftrightarrow y=f(x) \\
& \Leftrightarrow \mu_{x}\left(\left\{z:\left(\pi_{1} \circ g_{x} \circ \rho\right)(z)=(x, y)\right\}\right)=1,
\end{aligned}
$$

so $G_{f}$ is Borel, by Kechris [21], 17.25, thus $f$ is Borel. 
Denote by FBU the set of all trees $T$ for which $[T]$ has a full Borel uniformization:

$$
\begin{aligned}
\mathrm{FBU}= & \{T: T \text { is a tree on } \mathbb{N} \times \mathbb{N} \& \\
& {[T] \text { has a full Borel uniformization }\} . }
\end{aligned}
$$

We can identify here a tree with its characteristic function, a member of $2^{\mathbb{N}^{<N} \times \mathbb{N}^{<N}}$, which we can simply identify with $2^{\mathbb{N}}$. Clearly the set of trees is Borel. Since the set $E \subseteq 2^{\mathbb{N}} \times\left(\mathbb{N}^{\mathbb{N}}\right)^{2}$ given by

$$
(T, x, y) \in E \Leftrightarrow T \text { is a tree and }(x, y) \in E_{T}
$$

is clearly Borel, it follows that there is a Borel function $p$ such that for each tree $T, p(T) \in D$ and

$$
E_{T}=D_{p(T)} .
$$

So by $5.2, p^{-1}\left(\mathcal{E}_{\leq}^{*}\right)=p^{-1}\left(\mathcal{E}_{\sim}^{*}\right)=\mathrm{FBU}$; thus in order to show that $\mathcal{E}_{\leq}^{*}, \mathcal{E}_{\sim}^{*}$ are $\boldsymbol{\Sigma}_{2}^{1}$-complete it is enough to show that FBU is $\boldsymbol{\Sigma}_{2}^{1}$-complete.

Following up a conversation with one of the authors, John Steel first found a proof of this using non-standard models. We give below a different proof based on effective descriptive set theory. We assume that the reader is familiar with this theory as exposed for example in Moschovakis [28.

Lemma 5.3. The set $\mathrm{FBU}$ is $\boldsymbol{\Sigma}_{\mathbf{2}}^{\mathbf{1}}$-complete.

Proof. It is enough to show that every $\Sigma_{2}^{1}$ subset of $\mathbb{N}^{\mathbb{N}}$ is of the form $f^{-1}(\mathrm{FBU})$ for some Borel $f$.

We will first need some simple facts about trees (see Kechris 21, Section 2). Let $S, T$ be trees on sets $A, B$, resp. We let

$$
S \preceq T \Leftrightarrow \text { there is a strictly monotone map }
$$

$$
\varphi: S \rightarrow T \text {. }
$$

Also let $S * T$ be the tree on $A \times B$ given by

$$
S * T=\left\{(s, t) \in A^{<\mathbb{N}} \times B^{<\mathbb{N}}: \operatorname{length}(s)=\operatorname{length}(t) \& s \in S \& t \in T\right\} .
$$

Then, recalling that a tree is called illfounded if it has an infinite branch, we clearly have that $S * T$ is illfounded iff both $S, T$ are illfounded, so $S * T$ is wellfounded (i.e., not illfounded) iff at least one of $S, T$ is wellfounded. Moreover, if $S$ is wellfounded but $T$ is illfounded, then clearly $S \preceq S * T$. To see this, let $x \in[T]$ be an infinite branch of $T$ and define $\varphi(s)=(s, x \mid$ length $(s))$. Finally, recall that the set WF for all wellfounded trees is $\Pi_{1}^{1}$-complete and that the map

$$
\begin{gathered}
\rho: \mathrm{WF} \rightarrow \omega_{1} \\
\left(\omega_{1}=\right.\text { the first uncountable ordinal) given by } \\
\rho(S)=\text { the rank of } S
\end{gathered}
$$

is a $\Pi_{1}^{1}$-rank (also called $\Pi_{1}^{1}$-norm) on WF. Note also that for $T \in \mathrm{WF}, S \preceq T \Leftrightarrow$ $S \in \mathrm{WF} \& \rho(S) \leq \rho(T)$.

Now fix a $\Sigma_{2}^{1}$ set $A \subseteq \mathbb{N}^{\mathbb{N}}$ and let $B \subseteq\left(\mathbb{N}^{\mathbb{N}}\right)^{2}$ be $\Pi_{1}^{1}$ so that $u \in A \Leftrightarrow \exists v(u, v) \in B$ and let $g:\left(\mathbb{N}^{\mathbb{N}}\right)^{2} \rightarrow \mathbb{N}^{\mathbb{N}}$ be recursive so that $g(u, v)$ is a tree on $\mathbb{N}$, for each $u, v$, and $(u, v) \in B \Leftrightarrow g(u, v) \in \mathrm{WF}$. Thus

$$
\begin{aligned}
u \in A & \Leftrightarrow \exists v(g(u, v) \in \mathrm{WF}) \\
& \Leftrightarrow \exists T(T \in \mathrm{WF} \& \exists \varphi: g(u, v) \rightarrow T, \varphi \text { strictly monotone }) .
\end{aligned}
$$


Since $\left\{(x, y): y \in \Delta_{1}^{1}(x)\right\}$ is $\Pi_{1}^{1}$, let $f$ also be a recursive function such that $f(x, y)$ is a tree on $\mathbb{N}$ for all $x, y$ and

$$
y \in \Delta_{1}^{1}(x) \Leftrightarrow f(x, y) \in \mathrm{WF} .
$$

Now define $R \subseteq\left(\mathbb{N}^{\mathbb{N}}\right)^{3}$ by

$$
(u, x,\langle y, T, v, \varphi\rangle) \in R \Leftrightarrow \varphi: g(u, v) \rightarrow f(x, y) * T \text { is strictly monotone, }
$$

where \langle\rangle is an appropriate recursive coding of the objects indicated by a member of $\mathbb{N}^{\mathbb{N}}$. It is clear that $R$ is closed in $\left(\mathbb{N}^{\mathbb{N}}\right)^{3}$, so it is of the form $R=[U]=$ $\left\{(x, y, z) \in\left(\mathbb{N}^{\mathbb{N}}\right)^{3}: \forall n(x|n, y| n, z \mid n) \in U\right\}$, for some tree $U$ on $\mathbb{N} \times \mathbb{N} \times \mathbb{N}$. Then if $f(u)=U(u)=\{(s, t)$ : length $(s)=$ length $(t) \&(u \mid$ length $(s), s, t) \in U\}, f$ is a Borel map and $R_{u}=[f(u)]$. We show that

$$
\begin{aligned}
u \in A & \Leftrightarrow R_{u} \text { has a full Borel uniformization } \\
& \Leftrightarrow f(u) \in \mathrm{FBU},
\end{aligned}
$$

which shows that $A=f^{-1}(\mathrm{FBU})$.

Recall that a closed set $C \subseteq\left(\mathbb{N}^{\mathbb{N}}\right)^{2}$ admits a full Borel uniformization iff there is a parameter $p \in \mathbb{N}^{\mathbb{N}}$ such that for every $x$ there is $y \in \Delta_{1}^{1}(x, p)$ with $(x, y) \in C$ (Moschovakis [28, 4D.4). So it is enough to show that:

$$
u \in A \Leftrightarrow \exists p \forall x \exists\langle y, T, v, \varphi\rangle \in \Delta_{1}^{1}(x, p) R(u, x,\langle y, T, v, \varphi\rangle) .
$$

$\Leftarrow$ : Fix such a $p$. Then take $x=p$ and find $y, T, v, \varphi \in \Delta_{1}^{1}(x, p)=\Delta_{1}^{1}(x)$ such that $\varphi: g(u, v) \rightarrow f(x, y) * T$ is strictly monotone. As $y \in \Delta_{1}^{1}(x), f(x, y) \in \mathrm{WF}$, so $f(x, y) * T \in \mathrm{WF}$, so $g(u, v) \in \mathrm{WF}$ and thus $u \in A$.

$\Rightarrow$ : Let $T_{0}$ be a fixed recursive illfounded tree, e.g., $T_{0}=\mathbb{N}<\mathbb{N}$. Let $u_{0} \in A$. Pick $v_{0}$ so that $g\left(u_{0}, v_{0}\right)=S_{0} \in \mathrm{WF}$. Put $p=\left\langle u_{0}, v_{0}\right\rangle$ (so also $S_{0}$ is recursive in $p$ ). Given any $x$ we now have to find $\langle y, T, v, \varphi\rangle \in \Delta_{1}^{1}(x, p)$ satisfying $R\left(u_{0},\langle y, T, v, \varphi\rangle\right)$. We take $v=v_{0} \in \Delta_{1}^{1}(x, p)$. To find $y, T, \varphi$ we consider two cases:

(i) $\omega_{1}^{x}>\rho\left(S_{0}\right)$ (where $\omega_{1}^{x}$ is the first ordinal not recursive in $x$ ).

Then let $S_{0}^{*}$ be a wellfounded tree recursive in $x$ with $\rho\left(S_{0}\right)=\rho\left(S_{0}^{*}\right)$. It follows that there must be some $y \in \Delta_{1}^{1}(x) \subseteq \Delta_{1}^{1}(x, p)$ such that $\rho\left(S_{0}\right)=\rho\left(S_{0}^{*}\right) \leq$ $\rho(f(x, y))$, since otherwise

$$
\forall y\left(y \in \Delta_{1}^{1}(x) \Leftrightarrow \rho(f(x, y))<\rho\left(S_{0}^{*}\right)\right),
$$

so that $\left\{y: y \in \Delta_{1}^{1}(x)\right\}$ is $\Delta_{1}^{1}(x)$, a contradiction (Moschovakis [28], 4D.16). Therefore there is also some $\varphi_{1}: S_{0} \rightarrow f(x, y)$ strictly monotone such that $\varphi_{1} \in$ $\Delta_{1}^{1}\left(x, y, S_{0}\right) \subseteq \Delta_{1}^{1}(x, p)$ and this immediately gives some $\varphi: g\left(u_{0}, v\right)\left(=S_{0}\right) \rightarrow$ $f(x, y) * T_{0}, \varphi \in \Delta_{1}^{1}(x, p)$, which is strictly monotone. Thus if we choose $T=$ $T_{0},\langle y, T, v, \varphi\rangle$ works.

(ii) $\omega_{1}^{x} \leq \rho\left(S_{0}\right)$.

Then we claim that we can find $y \notin \Delta_{1}^{1}(x)$ such that $\mathcal{O}^{x, y} \in \Delta_{1}^{1}(x, p)$, where $\mathcal{O}^{a}$ is the Kleene $\mathcal{O}$ relative to $a$, i.e., the complete $\Pi_{1}^{1}(a)$ subset of $\mathbb{N}$.

Granting this claim, $f(x, y)$ is illfounded and we pick a branch $b \in[f(x, y)]$, which is recursive in $\mathcal{O}^{x, y}$, thus $b \in \Delta_{1}^{1}(x, p)$. Then let $\varphi(s)=(b \mid$ length $(s), s)$ for $s \in g\left(u_{0}, v\right)$. Clearly $\varphi \in \Delta_{1}^{1}(x, p)$ and $\varphi: g\left(u_{0}, v\right)\left(=S_{0}\right) \rightarrow f(x, y) * S_{0}$ is strictly monotone. Thus if we now choose $T=S_{0},\langle y, T, v, \varphi\rangle$ works.

It remains to prove the claim: Look at the set

$$
\left\{y: \omega_{1}^{x, y}=\omega_{1}^{x} \& y \notin \Delta_{1}^{1}(x)\right) .
$$


Since $\omega_{1}^{x} \leq \rho\left(S_{0}\right)<\omega_{1}^{S_{0}, x}$ we have that $\mathcal{O}^{x} \in \Delta_{1}^{1}\left(S_{0}, x\right)$, so this set is $\Delta_{1}^{1}\left(S_{0}, x\right)$. It is also comeager, so it has an element $y \in \Delta_{1}^{1}\left(S_{0}, x\right)$ (Moschovakis [28], 4F.20). Then as $\omega_{1}^{x, y}<\omega_{1}^{S_{0}, x}=\omega_{1}^{S_{0}, x, y}$, we have that $\mathcal{O}^{x, y} \in \Delta_{1}^{1}\left(S_{0}, x, y\right) \subseteq \Delta_{1}^{1}\left(S_{0}, x\right) \subseteq \Delta_{1}^{1}(x, p)$, so we are done.

Now let us say that a closed set $F \subseteq\left(\mathbb{N}^{\mathbb{N}}\right)^{2}$ has full projection if for every $x$ there is some $y$ with $(x, y) \in F$. By the Jankov, von Neumann Uniformization Theorem (see Kechris [21], 18.1), this is equivalent to saying the $F$ admits a full $\sigma\left(\boldsymbol{\Sigma}_{\mathbf{1}}^{\mathbf{1}}\right)$-measurable uniformization, where $\sigma\left(\boldsymbol{\Sigma}_{\mathbf{1}}^{\mathbf{1}}\right)$ is the smallest $\sigma$-algebra generated by the analytic sets.

Then by inspecting the argument in 5.2, we see the following, where $E \leq_{\sigma\left(\boldsymbol{\Sigma}_{1}^{1}\right)} F$ means that $E$ can be reduced to $F$ via a $\sigma\left(\boldsymbol{\Sigma}_{\mathbf{1}}^{\mathbf{1}}\right)$-measurable function, and similarly for $\sim_{\sigma\left(\Sigma_{1}^{1}\right)}$.

Lemma 5.4. For each tree $T$ we have

$$
\begin{aligned}
E^{*} \leq_{\sigma\left(\boldsymbol{\Sigma}_{1}^{1}\right)} E_{T} & \Leftrightarrow E^{*} \sim_{\sigma\left(\boldsymbol{\Sigma}_{1}^{1}\right)} E_{T} \\
& \Leftrightarrow[T] \text { has full projection } .
\end{aligned}
$$

Let

$$
\begin{aligned}
\mathrm{FP}= & \{T: T \text { is a tree on } \mathbb{N} \times \mathbb{N} \& \\
& {[T] \text { has full projection }\} . }
\end{aligned}
$$

Then it is quite standard that $\mathrm{FP}$ is a $\boldsymbol{\Pi}_{\mathbf{2}}^{\mathbf{1}}$-complete set: If $A \subseteq \mathbb{N}^{\mathbb{N}}$ is $\boldsymbol{\Pi}_{\mathbf{2}}^{\mathbf{1}}$, then $x \in A \Leftrightarrow \forall y(x, y) \in B$, where $B$ is $\boldsymbol{\Sigma}_{\mathbf{1}}^{\mathbf{1}}$, so $(x, y) \in B \Leftrightarrow \exists z(x, y, z) \in F$, where $F$ is closed in $\left(\mathbb{N}^{\mathbb{N}}\right)^{3}$, thus $F=[U]$, for some tree $U$ on $\mathbb{N} \times \mathbb{N} \times \mathbb{N}$. For each $x \in \mathbb{N}^{\mathbb{N}}$, let

$$
U(x)=\{(s, t) \mid \operatorname{length}(s)=\operatorname{length}(t) \text { and }(x \mid \operatorname{length}(s), s, t) \in U\} .
$$

Then $x \in A \Leftrightarrow U(x) \in \mathrm{FP}$, and clearly $x \mapsto U(x)$ is Borel.

We have FBU $\subseteq$ FP and since the first set is $\boldsymbol{\Sigma}_{\mathbf{2}}^{\mathbf{1}}$-complete while the second is $\mathbf{\Pi}_{\mathbf{2}}^{\mathbf{1}}$-complete, they are clearly not equal, i.e., FBU $\varsubsetneqq \mathrm{FP}$. (This is a well-known fact; see, e.g., Kechris [21], 18.17, but the argument used here seems to be new.) In particular, it follows trivially that there is a tree $T$ such that $E^{*} \leq_{\sigma\left(\boldsymbol{\Sigma}_{1}^{1}\right)} E_{T}$ but $E^{*} \not E_{B} E_{T}$. So we have:

Theorem 5.5. There is a pair of countable Borel equivalence relations $E, F$ such that $E \leq_{\sigma\left(\boldsymbol{\Sigma}_{1}^{1}\right)} F$ but $E \mathbb{\leq}_{B} F$ (and we moreover have that $F \leq_{B} E$ ).

\section{Finite RANK TORSION-FREE ABELIAN GROUPS}

Let $\cong_{n}$ be the isomorphism relation on torsion-free abelian groups of rank $\leq n$. These are exactly the groups isomorphic to subgroups of $\left(\mathbb{Q}^{n},+\right)$. Let us also denote by $\cong_{n}^{*}$ the restriction of $\cong_{n}$ to the rigid groups, where we call an abelian group rigid if its only automorphisms are $x \mapsto x, x \mapsto-x$.

It follows from a result of Baer (see, e.g., Fuchs [10]) that $\left(\cong_{1}\right) \sim_{B}\left(\cong_{1}^{*}\right) \sim_{B} E_{0}$, where $E_{0}$ is the equivalence relation on $2^{\mathbb{N}}$ defined by: $x E_{0} y \Leftrightarrow \exists n \forall m \geq n\left(x_{m}=\right.$ $\left.y_{m}\right)$. Hjorth [15] has shown that $E_{0}<_{B}\left(\cong_{n}\right)$ for all $n \geq 2$ and Thomas [33] extended this to show that $E_{0}<_{B}\left(\cong_{n}^{*}\right)$ for $n \geq 2$. Also Hjorth [15] showed that for $n \geq 3, \cong_{n}$ is not essentially treeable, i.e., there is no treeable countable Borel equivalence relation $E$ with $\left(\cong_{n}\right) \sim_{B} E$, and Thomas [33] extended this to $\cong_{n}^{*}$, for $n \geq 3$. Recently Kechris showed that $\cong_{n}$ is not essentially treeable for all $n \geq 2$. This is still open for $\cong_{2}^{*}$. (Recall that a countable Borel equivalence relation $E$ on 
$X$ is treeable if there is a Borel acyclic graph on $X$ whose connected components are the $E$-classes.) We show here the following, which in particular implies that no $\cong_{n}^{*}$ is universal.

Theorem 6.1. For any $n \geq 1,\left(\cong_{n}^{*}\right)<_{B}\left(\cong_{n+1}^{*}\right)$.

Proof. Let $S\left(\mathbb{Q}^{n}\right)$ be the space of all subgroups of $\mathbb{Q}^{n}$ (a closed subset of the space of all subsets of $\left.\mathbb{Q}^{n}\right)$. Since every isomorphism between subgroups of $\mathbb{Q}^{n}$ is induced by a matrix in $\mathrm{GL}_{n}(\mathbb{Q})$, we can view, up to $\sim_{B}, \cong_{n}$ as being the equivalence relation on $S\left(\mathbb{Q}^{n}\right)$ induced by the canonical action of $\mathrm{GL}_{n}(\mathbb{Q})$.

Let $Z_{n} \subseteq S\left(\mathbb{Q}^{n}\right)$ consist of all subgroups containing $\mathbb{Z}^{n}$. Then $\mathrm{SL}_{n}(\mathbb{Z})$ acts on $Z_{n}$ and Hjorth [15] shows that there is a probability measure $\nu_{n}$ on $Z_{n}$ invariant under this action with the following properties:

(i) The equivalence relation on $Z_{n}$ induced by this action is not tame, even on any set of $\nu_{n}$-measure 1 .

(ii) (Thomas 33]) There is a Borel set of $\nu_{n}$-measure $1, Y_{n}$, such that every element of $Y_{n}$ is rigid.

Now consider the ergodic decomposition of the action of $\mathrm{SL}_{n}(\mathbb{Z})$ on $Z_{n}$, as in Varadarajan 34. More precisely, let $\mathcal{E}$ be the standard Borel space of ergodic, invariant measures of this action. Then there is a Borel surjection $\pi: Z_{n} \rightarrow \mathcal{E}$, which is $\mathrm{SL}_{n}(\mathbb{Z})$-invariant, such that if $X_{e}=\left\{x \in Z_{n}: \pi(x)=e\right\}$, then $e\left(X_{e}\right)=1, \forall e \in \mathcal{E}$, and for any Borel set $A \subseteq Z_{n}$, and any $\mathrm{SL}_{n}(\mathbb{Z})$-invariant probability measure, $\nu$, we have $\nu(A)=\int e(A) d \nu_{*}(e)$, where $\nu_{*}=\pi_{*} \nu$ is the measure on $\mathcal{E}$ defined by $\nu_{*}(B)=\nu\left(\pi^{-1}(B)\right)$.

Since $\nu_{n}\left(Y_{n}\right)=1$, it follows that there is a Borel set $\mathcal{E}_{1} \subseteq \mathcal{E}$ such that $\left(\nu_{n}\right)_{*}\left(\mathcal{E}_{1}\right)=$ 1 and for $e \in \mathcal{E}_{1}, e\left(Y_{n}\right)=1$. Then there must be some $e_{1} \in \mathcal{E}_{1}$ which is non-atomic, since otherwise for each $e \in \mathcal{E}_{1}$, there is a unique orbit of $\operatorname{SL}_{n}(\mathbb{Z})$, say $\theta_{e}$, with $e\left(\theta_{e}\right)=1$, and then the set $W=\left\{x \in Z_{n}: \pi(x) \in \mathcal{E}_{1} \& x \in \theta_{\pi(x)}\right\}=\left\{x \in Z_{n}\right.$ : $\left.\pi(x) \in \mathcal{E}_{1} \& \pi(x)\left(\mathrm{SL}_{n}(\mathbb{Z}) \cdot x\right)=1\right\}$ is Borel, $\mathrm{SL}_{n}(\mathbb{Z})$-invariant, and the action of $\mathrm{SL}_{n}(\mathbb{Z})$ on $W$ is tame, as for $x, y \in W, y \in \mathrm{SL}_{n}(\mathbb{Z}) \cdot x \Leftrightarrow \pi(y)=\pi(x)$. But clearly $\nu_{n}(W)=1$, which is a contradiction.

So we can assume that there is an ergodic, non-atomic, $\mathrm{SL}_{n}(\mathbb{Z})$-invariant measure $\mu_{n}\left(=e_{1}\right)$ on $Z_{n}$ and there is a Borel $\mathrm{SL}_{n}(\mathbb{Z})$-invariant set $X_{n}\left(=X_{e_{1}} \cap Y_{n}\right) \subseteq Z_{n}$ with $\mu_{n}\left(X_{n}\right)=1$, such that every element of $X_{n}$ is rigid.

It is clear that $\left(\cong_{n}^{*}\right) \leq_{B}\left(\cong_{n+1}^{*}\right)$ and Thomas' result shows that $\left(\cong_{1}^{*}\right)<_{B}\left(\cong_{2}^{*}\right)$. So assume $n \geq 2$ and $\left(\cong_{n+1}^{*}\right) \leq_{B}\left(\cong_{n}^{*}\right)$, towards a contradiction. Say $f$ is a Borel function reducing $\cong_{n+1}^{*}$ to $\cong_{n}^{*}$. In particular, for $x, y \in X_{n+1}$ we have

$$
x E_{\mathrm{SL}_{n+1}(\mathbb{Z})}^{X_{n+1}} y \Rightarrow f(x) \cong_{n}^{*} f(y) .
$$

By ergodicity, there is $m \leq n$ and a set of $\mu_{n+1}$-measure 1 , so that for $x$ in that set $f(x)$ actually has rank exactly $m$, so that we can actually assume (by shrinking $X_{n+1}$ a bit if necessary) that for $x \in X_{n+1}, f(x) \in S\left(\mathbb{Q}^{m}\right), f(x)$ has rank $m$, and

$$
x E_{\mathrm{SL}_{n+1}(\mathbb{Z})}^{X_{n+1}} y \Rightarrow f(x) \cong{ }_{m}^{*} f(y) \text {. }
$$

It follows that there is a strict Borel cocycle $\alpha: \mathrm{SL}_{n+1}(\mathbb{Z}) \times X_{n+1} \rightarrow A^{m}(\mathbb{Q})$, where $A^{m}=\mathrm{GL}_{m} /\left\{I_{m},-I_{m}\right\}$, with $I_{m}=$ the identity $m \times m$ matrix, such that

$$
\alpha(g, x)=\left[\text { the unique } h \in A^{m}(\mathbb{Q}) \text { such that } h \cdot f(x)=f(g \cdot x)\right] .
$$

Now $|\operatorname{det}(\alpha)|$ is also a strict Borel cocycle, $|\operatorname{det}(\alpha)|: \mathrm{SL}_{n+1}(\mathbb{Z}) \times X_{n+1} \rightarrow \mathbb{Q}_{+}^{*}(=$ the multiplicative group of the positive rationals), so since $\mathbb{Q}_{+}^{*}$ is amenable and 
$\mathrm{SL}_{n+1}(\mathbb{Z})$ is Kazhdan (see Zimmer [35, 4.1.2, and de la Harpe and Valette [3, 3.a.5, page 34 , resp.), it follows by 3.1 that there is a Borel cocycle $\delta: \mathrm{SL}_{n+1}(\mathbb{Z}) \times X_{n+1} \rightarrow$ $\mathbb{Q}_{+}^{*}$ taking values in a finite subgroup of $\mathbb{Q}_{+}^{*}$, thus into $\{1\}$, such that $|\operatorname{det}(\alpha)| \sim \delta$. So there is Borel $F: X_{n+1} \rightarrow \mathbb{Q}_{+}^{*}$ with $|\operatorname{det}(\alpha(g, x))|=F(g \cdot x) F(x)^{-1} \mu_{n+1}$-a.e.

Choose for each $a \in \mathbb{Q}_{+}^{*}$ an element $\bar{a} \in A^{m}(\mathbb{Q})$ with $|\operatorname{det}(\bar{a})|=a$ and let $\bar{F}: X_{n+1} \rightarrow A^{m}(\mathbb{Q})$ be defined by $\bar{F}(x)=\overline{F(x)}$. Then if

$$
\bar{\alpha}(g, x)=(\bar{F}(g \cdot x))^{-1} \alpha(g, x) \bar{F}(x),
$$

$\bar{\alpha}$ is a Borel cocycle from $\mathrm{SL}_{n+1}(\mathbb{Z}) \times X_{n+1}$ into $A^{m}(\mathbb{Q}), \alpha \sim \bar{\alpha}$, and as

$$
\begin{aligned}
|\operatorname{det}(\bar{\alpha}(g, x))| & =\left|\operatorname{det}(\bar{F}(g \cdot x))^{-1}\right| \cdot|\operatorname{det}(\alpha(g, x))| \cdot|\operatorname{det}(\bar{F}(x))| \\
& =F(g \cdot x)^{-1} \cdot|\operatorname{det}(\alpha(g, x))| \cdot F(x)=1 \quad \mu_{n+1} \text {-a.e., }
\end{aligned}
$$

we conclude that $\bar{\alpha}(g, x) \in H^{m}(\mathbb{Q}), \mu_{n+1}$-a.e., with $H^{m}=\mathrm{SL}_{m}^{ \pm} /\left\{I_{m},-I_{m}\right\} \subseteq A^{m}$, where $\mathrm{SL}_{m}^{ \pm}$consists of all $m \times m$ matrices with determinant \pm 1 . Now $\mathrm{SL}_{n+1}(\mathbb{Z})$ is a Kazhdan group, so by 3.2 we have that $\bar{\alpha} \sim \beta$, where $\beta\left(\mathrm{SL}_{n+1}(\mathbb{Z}) \times X_{n+1}\right) \subseteq \Lambda$, a finitely generated subgroup of $H^{m}(\mathbb{Q})$, and we can assume that $\beta$ is a strict cocycle. So there is a finite set of primes $p_{1}, \cdots, p_{n}$ such that $\Lambda \subseteq H^{m}\left(\mathbb{Z}\left[1 / p_{1}, \cdots, 1 / p_{n}\right]\right)$.

Now $H^{m}\left(\mathbb{Z}\left[1 / p_{1}, \cdots, 1 / p_{n}\right]\right)$ is a discrete subgroup of $H^{m}(\mathbb{R}) \times H^{m}\left(\mathbb{Q}_{p_{1}}\right) \times \cdots \times$ $H^{m}\left(\mathbb{Q}_{p_{n}}\right)$ (see the corresponding argument in the proof of 4.5) and $\mathrm{SL}_{n+1}(\mathbb{Z})$ is a lattice in $\mathrm{SL}_{n+1}(\mathbb{R})$ (see Zimmer [35], page 1, Example (c)).

Consider the action of $\mathrm{SL}_{n+1}(\mathbb{Z})$ on $X_{n+1}$ and the cocycle $\beta: \mathrm{SL}_{n+1}(\mathbb{Z}) \times X_{n+1} \rightarrow$ $H^{m}\left(\mathbb{Z}\left[1 / p_{1}, \cdots, 1 / p_{n}\right]\right)$, and using Section 2 (paragraph following 2.2 ) consider the induced Borel action of $\mathrm{SL}_{n+1}(\mathbb{R})$ on $Y$ (which also has an invariant, ergodic measure) and the induced cocycle $\hat{\beta}: \mathrm{SL}_{n+1}(\mathbb{R}) \times Y \rightarrow H^{m}\left(\mathbb{Z}\left[1 / p_{1}, \cdots, 1 / p_{n}\right]\right)$. Viewing $\hat{\beta}$ as a cocycle into $H^{m}(\mathbb{R}) \times H^{m}\left(\mathbb{Q}_{p_{1}}\right) \times \cdots \times H^{m}\left(\mathbb{Q}_{p_{m}}\right)$ and letting $\pi$ be the projection of this product to one of its factors, consider the cocycle $\pi \circ \hat{\beta}$. By $3.8, \pi \circ \hat{\beta}$ is equivalent to a cocycle with values in a compact subgroup, so this shows that $\hat{\beta} \sim \gamma_{1}$, where $\gamma_{1}$ is a cocycle with values into a compact subgroup, so, by $2.4, \hat{\beta}$ is equivalent to a cocycle with values in a finite subgroup of $H^{m}\left(\mathbb{Z}\left[1 / p_{1}, \cdots, 1 / p_{n}\right]\right)$ and thus, by $2.3, \beta$ is equivalent to a cocycle $\gamma$ with values in a finite subgroup of $H^{m}\left(\mathbb{Z}\left[1 / p_{1}, \cdots, 1 / p_{n}\right]\right) \subseteq A^{m}(\mathbb{Q})$. Since $\alpha \sim \bar{\alpha} \sim \beta \sim \gamma$, we have $\alpha \sim \gamma$ as well. Say $\alpha(g, x)=B(g \cdot x) \gamma(g, x) B(x)^{-1}, \mu_{n+1}$-a.e., $B: X_{n+1} \rightarrow A^{m}(\mathbb{Q})$ a Borel function. Then let $M \subseteq X_{n+1}$ be a Borel set of positive $\mu_{n+1}$-measure on which $B$ is constant. It follows that $\phi(x)=\left\{f(y): y \in M \& y E_{\mathrm{SL}_{n+1}(\mathbb{Z})}^{X_{n+1}} x\right\}$ is finite, for all $x \in M$. By ergodicity, $\phi(x)$ is fixed on a Borel set $M_{0} \subseteq M$ of positive $\mu_{n+1^{-}}$ measure, and so if $M_{1}=\mathrm{SL}_{n+1}(\mathbb{Z}) \cdot M_{0}$, it follows that $\mu_{n+1}\left(M_{1}\right)=1$ and $f\left(M_{1}\right)$ is contained in a single $\cong_{m}^{*}$-class. But since $f$ is a reduction, this means that $M_{1}$ is contained in a single $\cong_{n+1}^{*}$-class, i.e., in a single $\mathrm{GL}_{n+1}(\mathbb{Q})$ orbit, so it is countable, violating the non-atomicity of $\mu_{n+1}$.

\section{Miscellanea}

We mention here some other results that can be proved by the methods used in this paper.

(i) Consider the canonical action of $\mathrm{GL}_{n}(\mathbb{Z})$ on $\mathbb{T}^{n}=(\mathbb{R} / \mathbb{Z})^{n}$ and denote by $R_{n}$ the corresponding equivalence relation. Then we show (Theorem 7.1) that $m<n \Rightarrow R_{n} \not_{B} R_{m}$. (In fact, in Theorem 7.1, we will prove a more general result concerning "essential" Borel reducibility, defined below.) We also show that $m \leq n \Rightarrow R_{m} \leq_{B} R_{n}$ (Theorem 7.2), therefore, $m<n$ implies that $R_{m}<_{B} R_{n}$. 
On the other hand, consider the canonical action of $\mathrm{SL}_{n}(\mathbb{Z})$ on $\mathbb{T}^{n}=(\mathbb{R} / \mathbb{Z})^{n}$ and denote by $R_{n}^{\prime}$ the corresponding equivalence relation. It should be pointed out here that we do not know whether, in general, $m<n \Rightarrow R_{m}^{\prime}<_{B} R_{n}^{\prime}$.

If $R$ is a countable Borel equivalence relation on a standard Borel space $X$, if $\mu$ is a measure on $X$, and if $S$ is a countable equivalence relation on a standard Borel space $Y$, then we say that $R$ is essentially reducible to $S$ if there is a conull Borel subset $X_{0} \subseteq X$ such that $R \mid X_{0} \leq_{B} S$. This will be denoted $R \leq_{B} S$ (a.e.). The notation $R \mathbb{L}_{B} S$ (a.e.) will mean that $R$ is not essentially reducible to $S$, i.e., that, for any conull Borel subset $X_{0} \subseteq X$, we have $R \mid X_{0} \mathbb{L}_{B} S$.

For all integers $v \geq 1$, let $\mathbb{Z}_{v}:=(1 / v) \mathbb{Z}=\{i / v: i \in \mathbb{Z}\} \subseteq \mathbb{Q}$. For all integers $l, v \geq 1$, let $R_{l v}$ be the equivalence relation defined by the orbits of the action of $\mathrm{GL}_{l}(\mathbb{Z})$ on $\left(\mathbb{R} / \mathbb{Z}_{v}\right)^{l}$.

Theorem 7.1. For all integers $m, n, s, t \geq 1: m<n \Rightarrow R_{n t} \mathbb{L}_{B} R_{m s}$ (a.e.).

Proof. The proof is by induction on $m$, and we may assume $m \geq 2$. Fix integers $n, s, t \geq 1$ and assume that $R_{n t} \leq_{B} R_{m s}$ (a.e.). We aim for a contradiction.

Let $G:=\mathrm{GL}_{m}(\mathbb{Z}), R:=R_{m s}$ and $S:=R_{n t}$. Then $S \leq_{B} R$ (a.e.). Let $A:=$ $\left(\mathbb{R} / \mathbb{Z}_{s}\right)^{m}$ and let $B:=\left(\mathbb{R} / \mathbb{Z}_{t}\right)^{n}$. We denote the action of $G$ on $A$ by $(g, a) \mapsto$ $g a: G \times A \rightarrow A$. Let $A^{\prime}:=\left\{a \in A: \exists g \in G \backslash\left\{1_{G}\right\}(g a=a)\right\}$ and let $\mu$ be the canonical measure for $S$. By the argument in 6.1 , we see that $S \not \leq_{B} \quad R \mid\left(A \backslash A^{\prime}\right)$ (a.e.). Ergodicity of $\mu$ then implies that $S \leq_{B} R \mid A^{\prime}$ (a.e.).

For any integer $d \geq 1$, for any $a=\left(a_{1}, \cdots, a_{d}\right) \in \mathbb{R}^{d}$, we will say that $a$ is totally irrational if $1, a_{1}, \cdots, a_{d}$ are linearly independent over $\mathbb{Q}$. For any integer $d \geq 1$, for any $a=\left(a_{1}, \cdots, a_{d}\right) \in \mathbb{R}^{d}$, we will say that $a$ is in canonical position if there exists $i \in\{0, \cdots, d\}$ such that $\left(a_{1}, \cdots, a_{i}\right) \in \mathbb{Q}^{i}$ and such that $\left(a_{i+1}, \cdots, a_{d}\right)$ is totally irrational. We call $i$ the index of $a$ and we call $\left(a_{1}, \cdots, a_{i}\right)$ the rational part of $a$. (Note: If $a \in \mathbb{Q}^{d}$, then $a$ is in canonical position, with index $d$ and rational part $a$. If $a$ is totally irrational, then $a$ is in canonical position, with index 0 and with no rational part.)

The conditions that $a$ be totally irrational or in canonical position are both invariant under translation by elements of $\mathbb{Q}^{d}$. It follows that, if $\Gamma$ is an additive subgroup of $\mathbb{Q}^{d}$ and if $a \in \mathbb{R}^{d} / \Gamma$ and if a preimage of $a$ in $\mathbb{R}^{d}$ is totally irrational (resp. in canonical position), then all preimages of $a$ in $\mathbb{R}^{d}$ are totally irrational (resp. in canonical position). In this case, we will say that $a$ is totally irrational (resp. in canonical position).

We claim, for all additive subgroups $\Gamma \subseteq \mathbb{Q}^{d}$, for all $a \in \mathbb{R}^{d} / \Gamma$, that there exists $g \in \mathrm{GL}_{d}(\mathbb{Z})$ such that $g a$ is in canonical position. Sketch of proof: It suffices to prove this when $\Gamma=\{0\}$. By induction on $d$, it suffices to show that if $a$ is not totally irrational, then, for some $g \in \mathrm{GL}_{d}(\mathbb{Z})$, the first coordinate of $g a$ is rational. However, if $a$ is not totally irrational, then there exists $k \in \mathbb{Z}^{d} \backslash\{0\}$ such that $k \cdot a \in \mathbb{Q}$, where $\cdot$ denotes the standard dot product. Dividing $k$ by the gcd of its entries, we may assume that $k$ is primitive (i.e., that the entries of $k$ are relatively prime). Then $k$ is the first vector in some base of the finitely generated abelian group $\mathbb{Z}^{d}$. So, making a change of base, we may replace $a$ by some element of $\left(\mathrm{GL}_{d}(\mathbb{Z})\right) a$ and $k$ by $(1,0, \cdots, 0)$. The first coordinate of $a$ is then $k \cdot a$, which is rational, as desired. The claim is proved.

For all $i \in\{1, \cdots, m\}$, let $Q_{i}:=\left(\mathbb{Q} / \mathbb{Z}_{s}\right)^{i}$. For all $i \in\{1, \cdots, m\}$, for all $q \in Q_{i}$, let $A_{i q}^{\prime}$ denote the collection of all $a \in A$ such that $a$ is in canonical position with 
index $i$ and with rational part $q$. Note, for all $q \in Q_{m}$, that we have $A_{m q}^{\prime}=\{q\}$, so $\left|A_{m q}^{\prime}\right|=1$. Let $A_{0}^{\prime}$ be the union of all $A_{i q}^{\prime}$ over $i \in\{1, \cdots, m\}$ and $q \in Q_{i}$.

Let $a \in A^{\prime}$. Then there is some $g \in G \backslash\left\{1_{G}\right\}$ such that $\left(g-1_{G}\right) a=0$. Then if $k$ is a non-zero row in $g-1_{G}$, we have $k \cdot a=0$, where $\cdot$ denotes the standard dot product. This proves that $a$ is not totally irrational. Choosing $g_{0} \in G$ such that $g_{0} a$ is in canonical position, we find that $g_{0} a$ is not totally irrational, so the index $i$ of $g_{0} a$ is not 0 . If $q$ is the rational part of $g_{0} a$, then we have $g_{0} a \in A_{i q}^{\prime}$, so $a \in g_{0}^{-1} A_{i q}^{\prime} \subseteq G A_{0}^{\prime}$. This argument proves that $A^{\prime} \subseteq G A_{0}^{\prime}$. Since $A^{\prime}$ is $G$-invariant and since $A_{0}^{\prime} \subseteq A^{\prime}$, it follows that $G A_{0}^{\prime} \subseteq A^{\prime}$. Then $A^{\prime}=G A_{0}^{\prime}$.

Then $R\left|A^{\prime} \leq_{B} R\right| A_{0}^{\prime}$, so, by ergodicity of $S$, fix $i \in\{1, \cdots, m\}$ and $q \in Q_{i}$ such that $S \leq_{B} R \mid A_{i q}^{\prime}$ (a.e.). Since $\mu$ is not atomic, we see that $\left|A_{i q}^{\prime}\right| \neq 1$, which implies that $i \neq m$, so $i \in\{1, \cdots, m-1\}$. Choose $q_{1}, \cdots, q_{i} \in \mathbb{Q} / \mathbb{Z}_{s}$ such that $q=\left(q_{1}, \cdots, q_{i}\right)$.

Let $U:=\{s, 2 s, 3 s, \cdots\}$. For all $u \in U$, let $p_{u}: \mathbb{R} / \mathbb{Z}_{s} \rightarrow \mathbb{R} / \mathbb{Z}_{u}$ be the canonical homomorphism. Choose $r \in U$ such that $\mathbb{Z} q_{1}+\cdots+\mathbb{Z} q_{i}=\operatorname{ker} p_{r}$. Let $j:=m-i$. Then $1 \leq j \leq m-1$.

Define $p:\left(\mathbb{R} / \mathbb{Z}_{s}\right)^{j} \rightarrow\left(\mathbb{R} / \mathbb{Z}_{r}\right)^{j}$ by $p\left(c_{1}, \cdots, c_{j}\right)=\left(p_{r}\left(c_{1}\right), \cdots, p_{r}\left(c_{j}\right)\right)$. Define $f: A_{i q}^{\prime} \rightarrow\left(\mathbb{R} / \mathbb{Z}_{r}\right)^{j}$ by $f\left(a_{1}, \cdots, a_{m}\right)=p\left(a_{i+1}, \cdots, a_{m}\right)$. It remains to prove that $f$ is a Borel reduction of $R_{m s} \mid A_{i q}^{\prime}$ to $R_{j r}$, whereupon we will have $S \leq_{B} R_{j r}$ (a.e.), contradicting the induction assumption.

So fix $a=\left(a_{1}, \cdots, a_{m}\right) \in A_{i q}^{\prime}$ and $b=\left(b_{1}, \cdots, b_{m}\right) \in A_{i q}^{\prime}$. Let $a_{0}:=f(a)$ and let $b_{0}:=f(b)$ so $a_{0}, b_{0} \in\left(\mathbb{R} / \mathbb{Z}_{r}\right)^{j}$. We wish to show:

$$
\exists g \in G(g a=b) \quad \text { iff } \quad \exists g_{0} \in \mathrm{GL}_{j}(\mathbb{Z})\left(g_{0} a_{0}=b_{0}\right) .
$$

Set $a^{\prime}=\left(a_{1}, \cdots, a_{i}\right)$ and $b^{\prime}:=\left(b_{1}, \cdots, b_{i}\right)$. Set $a^{\prime \prime}=\left(a_{i+1}, \cdots, a_{m}\right)$ and $b^{\prime \prime}:=$ $\left(b_{i+1}, \cdots, b_{m}\right)$. Then $a^{\prime}=q=b^{\prime}$. Moreover, $a^{\prime \prime}$ and $b^{\prime \prime}$ are totally irrational. Also, $a_{0}=p\left(a^{\prime \prime}\right)$ and $b_{0}=p\left(b^{\prime \prime}\right)$.

Proof of "if". By definition of $r$, we have ker $p=\mathbb{Z}^{j \times i} q$. So, since we have $p\left(b^{\prime \prime}-g_{0} a^{\prime \prime}\right)=b_{0}-g_{0} a_{0}=0$, choose $Z \in \mathbb{Z}^{j \times i}$ such that $b^{\prime \prime}-g_{0} a^{\prime \prime}=Z a^{\prime}$. Recall that $a^{\prime}=q=b^{\prime}$.

Let $I$ denote the $i \times i$ identity matrix and let 0 denote the $i \times j$ matrix with all entries equal to 0 . Let $g:=\left(\begin{array}{cc}I & 0 \\ Z & g_{0}\end{array}\right)$. Then $g a=b$. This concludes the proof of "if".

Proof of "only if". Choose $X \in \mathbb{Z}^{i \times i}, Y \in \mathbb{Z}^{i \times j}, Z \in \mathbb{Z}^{j \times i}$ and $g_{0} \in \mathbb{Z}^{j \times j}$ such that

$$
g=\left(\begin{array}{cc}
X & Y \\
Z & g_{0}
\end{array}\right) .
$$

Then $g a=b$ implies that $X a^{\prime}+Y a^{\prime \prime}=b^{\prime}$ and $Z a^{\prime}+g_{0} a^{\prime \prime}=b^{\prime \prime}$. We wish to show that $\operatorname{det} g_{0} \in\{ \pm 1\}$ and that $g_{0} a_{0}=b_{0}$.

We have $Y a^{\prime \prime}=b^{\prime}-X a^{\prime} \in Q_{i}+\mathbb{Z}^{i \times i} Q_{i} \subseteq Q_{i}$. On the other hand, $a^{\prime \prime}$ is totally irrational. It follows that $Y=0$. Then $\operatorname{det} g=(\operatorname{det} X)\left(\operatorname{det} g_{0}\right)$ and $\operatorname{det} X, \operatorname{det} g_{0} \in \mathbb{Z}$ and $\operatorname{det} g \in\{ \pm 1\}$. So $\operatorname{det} g_{0} \in\{ \pm 1\}$.

Define $\pi:\left(\mathbb{R} / \mathbb{Z}_{s}\right)^{i} \rightarrow\left(\mathbb{R} / \mathbb{Z}_{r}\right)^{i}$ by $\pi\left(c_{1}, \cdots, c_{i}\right)=\left(p_{r}\left(c_{1}\right), \cdots, p_{r}\left(c_{i}\right)\right)$. Then $b_{0}=p\left(b^{\prime \prime}\right)=p\left(Z a^{\prime}+g_{0} a^{\prime \prime}\right)=Z\left(\pi\left(a^{\prime}\right)\right)+g_{0}\left(p\left(a^{\prime \prime}\right)\right)$. Since $a^{\prime}=q$, it follows from the definition of $r$ that $\pi\left(a^{\prime}\right)=0$. Moreover, $p\left(a^{\prime \prime}\right)=a_{0}$. Then $b_{0}=g_{0} a_{0}$, as desired. This concludes the proof of "only if". 
Theorem 7.2. For all integers $m, n \geq 1: m \leq n \Rightarrow R_{m} \leq_{B} R_{n}$.

Proof. We will use the notation and terminology established in the proof of 7.1. It is of course enough to show that for $m \geq 1, R_{m} \leq_{B} R_{m+1}$.

We start by noticing that if $d \geq 1$, then $a \in(\mathbb{R} / \mathbb{Z})^{d}$ is in canonical position with index $i \in\{0, \cdots, d\}$ and $b \in(\mathbb{R} / \mathbb{Z})^{d}$ is in canonical position with index $j \in\{0, \cdots, d\}$, and if $i \neq j$, then $a, b$ are not in the same $\mathrm{GL}_{d}(\mathbb{Z})$-orbit.

Next we verify that if $\left(a_{1}, \cdots, a_{d}\right) \in(\mathbb{R} / \mathbb{Z})^{d}$ is in canonical position and has index $i \geq 1$, then there exists $g \in \mathrm{GL}_{d}(\mathbb{Z})$ with

$$
g a=\left(0, \cdots, 0, q, a_{i+1}, \cdots, a_{d}\right),
$$

for some $q \in \mathbb{Q} / \mathbb{Z}$. For that it is enough to check that if $i \geq 1$ and $\left(q_{1}, \cdots, q_{i}\right) \in$ $(\mathbb{Q} / \mathbb{Z})^{i}$, then there is $q \in \mathbb{Q} / \mathbb{Z}$ and $g \in \mathrm{GL}_{i}(\mathbb{Z})$ with

$$
g(0, \cdots, 0, q)=\left(q_{1}, \cdots, q_{i}\right) .
$$

To see this, notice that we can write $q_{\ell}=m_{\ell} r \bmod \mathbb{Z}$, where $\left(m_{1}, \cdots, m_{i}\right) \in \mathbb{Z}^{i}$ are relatively prime and $r \in \mathbb{Q}$, so there is $g \in \mathrm{GL}_{i}(\mathbb{Z})$ with last column equal to $\left(m_{1}, \cdots, m_{i}\right)$ (viewed as a column vector). If $q=r \bmod \mathbb{Z}$, then $g(0, \cdots, 0, q)=$ $\left(q_{1}, \cdots, q_{i}\right)$.

So for $0 \leq i \leq m$, let

$$
\begin{aligned}
& A_{m}^{i}=\left\{a \in(\mathbb{R} / \mathbb{Z})^{m}: a=\left(0, \cdots, q, a_{i+1}, \cdots, a_{m}\right),\right. \\
& \text { for some } q \in \mathbb{Q} / \mathbb{Z} \text { and }\left(a_{i+1}, \cdots, a_{m}\right) \\
&\text { totally irrational }\} .
\end{aligned}
$$

Then it is enough to show that

$$
R_{m}\left|A_{m}^{i} \leq_{B} R_{m+1}\right| A_{m+1}^{i+1} .
$$

We consider cases on $i \in\{0, \cdots, m\}$.

Case 1: $i=0$. Then $\left(a_{1}, \cdots, a_{m}\right) \in A_{m}^{0} \mapsto\left(0, a_{1}, \cdots, a_{m}\right) \in A_{m+1}^{1}$ is a reduction.

Case 2: $i>1$. Then again we claim that

$$
\left(a_{1}, \cdots, a_{m}\right) \in A_{m}^{1} \mapsto\left(0, a_{1}, \cdots, a_{m}\right) \in A_{m+1}^{i+1}
$$

is a reduction. The main point here is to observe that if $(0,0, \cdots, 0, q),(0,0, \cdots, 0, r)$ $\in(\mathbb{Q} / \mathbb{Z})^{i+1}$ are in the same $\mathrm{GL}_{i+1}(\mathbb{Z})$-orbit and $i>1$, then $q, r$ generate the same subgroup of $\mathbb{R} / \mathbb{Z}$, so $(0, \cdots, 0, q),(0, \cdots, 0, r) \in(\mathbb{Q} / \mathbb{Z})^{i}$ are in the same $\mathrm{GL}_{i}(\mathbb{Z})$ orbit.

Case 3: $i=1$. Fix a set of representatives $T=\left\{t_{j}: j=0,1,2, \cdots\right\}$ for the following equivalence relation on $\mathbb{Q} / \mathbb{Z}: q \sim q^{\prime}$ iff $q=q^{\prime}$ or $q=-q^{\prime}$. We agree that $t_{0}=0 \bmod \mathbb{Z}$. Every $\left(q, a_{2}, \cdots, a_{m}\right) \in A_{m}^{1}$ can be sent to some $\left(t_{i}, a_{2}, \cdots, a_{m}\right) \in$ $A_{m}^{1}$ by an element of $\mathrm{GL}_{m}(\mathbb{Z})$, so if

$$
B_{m}^{1}=\left\{\left(q, a_{2}, \cdots, a_{m}\right) \in A_{m}^{1}: q \in T\right\},
$$

it is enough to show that

$$
R_{m}\left|B_{m}^{1} \leq_{B} R_{m+1}\right| A_{m+1}^{2} .
$$

For each $j \geq 1$, choose $m_{j}, n_{j} \in \mathbb{Z}, n_{j} \neq 0$, relatively prime so that $t_{j}=\frac{m_{j}}{n_{j}}$ $\bmod \mathbb{Z}$. Then notice that by induction on $j \geq 1$, we can find $0 \neq k_{j} \in \mathbb{Z}$ such that 
$k_{j}, m_{j}$ are relatively prime and such that, if we put

$$
s_{j}=\frac{1}{k_{j} n_{j}} \quad \bmod \mathbb{Z} \quad \text { and } \quad u_{j}=\frac{m_{j}}{k_{j} n_{j}} \bmod \mathbb{Z},
$$

then for $\ell<j,\left(s_{j}, u_{j}\right),\left(s_{\ell}, u_{\ell}\right)$ are in distinct $\mathrm{GL}_{2}(\mathbb{Z})$-orbits. The main point here is that for $0 \neq k \in \mathbb{Z},\left(\frac{1}{k n_{j}} \bmod \mathbb{Z}, 0\right)$ is in the $\mathrm{GL}_{2}(\mathbb{Z})$-orbit of $\left(\frac{1}{k n_{j}} \bmod \mathbb{Z}, t_{j}\right)$, and for $k \neq k^{\prime},\left(\frac{1}{k n_{j}} \bmod \mathbb{Z}, 0\right)$ and $\left(\frac{1}{k^{\prime} n_{j}} \bmod \mathbb{Z}, 0\right)$ are in distinct $\mathrm{GL}_{2}(\mathbb{Z})$-orbits.

For each $k=1,2, \cdots$, fix a Borel map $x \mapsto x / / k: \mathbb{R} / \mathbb{Z} \rightarrow \mathbb{R} / \mathbb{Z}$ such that $k(x / / k)=x$. We are now ready to define our reduction: First map $\left(0, a_{2}, \cdots, a_{m}\right) \in$ $B_{m}^{1}$ to $\left(0,0, a_{2}, \cdots, a_{m}\right)$. Then, for each $j \geq 1, \operatorname{map}\left(t_{j}, a_{2}, \cdots, a_{m}\right) \in B_{m}^{1}$ to $\left(s_{j}, u_{j}, a_{2} / / k_{j}, \cdots, a_{m} / / k_{j}\right) \in A_{m+1}^{2}$.

(ii) Recall from the introduction that there is a universal countable Borel equivalence relation $E_{\infty}$. This equivalence relation has many manifestations, i.e., there are various examples of countable Borel equivalence relations $E$ with $E \sim_{B} E_{\infty}$. For example, the translation action of the free group with two generations $F_{2}$ acting on its subsets gives rise to a universal equivalence relation. It has been observed that there is no known free Borel action of a countable group which gives rise to a universal equivalence relation. So this led to the following open problem: Is there a countable group $\Gamma$ and a free Borel action of $\Gamma$ so that the corresponding equivalence relation is universal? We only remark that one can show by the methods used here that no $\Gamma$ which is (up to isomorphism) a discrete subgroup of some $\mathrm{GL}_{n}(\mathbb{C})$ or $\mathrm{GL}_{n}\left(\mathbb{Q}_{p}\right)$ can possibly work.

(iii) Finally, although in Section 5 we have computed the exact descriptive complexity of the equivalence relation $\mathcal{E}_{\sim}$ (of bi-reducibility of countable Borel equivalence relations), there are other questions that can be raised concerning the possible complexity of $\mathcal{E}_{\sim}$. For example, Theorem 4.1 implies that if $R$ is the equivalence relation on the set of codes $D$ of Borel sets, say of $2^{\mathbb{N}}$ (defined in a similar fashion as in Section 5), and $d \in D \mapsto D_{d}$ is the associated map (so that the Borel sets are exactly those of the form $D_{d}$, for $d \in D$ ), then the equivalence relation

$$
R(d, e) \Leftrightarrow D_{d}=D_{e}
$$

is Borel reducible to $\mathcal{E}_{\sim}$. From this it immediately follows that every Borel equivalence relation is Borel reducible to $\mathcal{E}_{\sim}$ and Gao 11 has extended this to show that every $\boldsymbol{\Sigma}_{1}^{1}$ equivalence relation is Borel reducible to $\mathcal{E}_{\sim}$. One can further ask here: Is every $\boldsymbol{\Pi}_{1}^{1}$ or even $\boldsymbol{\Sigma}_{\mathbf{2}}^{\mathbf{1}}$ equivalence relation Borel reducible to $\mathcal{E}_{\sim}$ ?

\section{ACKNOWLEDGMENT}

We would like to thank G. Hjorth for asking a question (related to Section 6) that triggered our investigations in this paper. We would also like to thank an anonymous referee for many detailed comments that helped improve the presentation.

\section{REFERENCES}

1. S. Adams, Indecomposability of treed equivalence relations, Israel J. Math., 86 (3), 362-380, 1988. MR 90m:28022

2. S. Adams, Trees and amenable equivalence relations, Erg. Th. and Dyn. Syst., 10, 1-14, 1990. MR 91d:28041 
3. P. de la Harpe and A. Valette, La Propriété (T) de Kazhdan pour les Groupes Localement Compacts, Astérisque, 175, Soc. Math. de France, 1989. MR 90m:22001

4. J. Dieudonné, Sur les Groupes Classiques, Hermann, Paris, 1973. MR 49:9094

5. R. Dougherty, S. Jackson, and A.S. Kechris, The structure of hyperfinite Borel equivalence relations, Trans. Amer. Math. Soc., 341 (1), 193-225, 1994. MR 94c:03066

6. E.G. Effros, Transformation groups and C*-algebras, Ann. of Math., 81, 38-55, 1965. MR 30:5175

7. J. Feldman, P. Hahn, and C.C. Moore, Orbit structure and countable sections for actions of continuous groups, Adv. in Math., 26, 186-230, 1979. MR 58:1121

8. J. Feldman and C.C. Moore, Ergodic equivalence relations and von Neumann algebras, I, Trans. Amer. Math. Soc., 234, 289-324, 1977. MR 58:28261a

9. H. Friedman and L. Stanley, A Borel reducibility theory for classes of countable structures, J. Symb. Logic, 54 (3), 894-914, 1989. MR 91f:03062

10. L. Fuchs, Infinite Abelian Groups, Academic Press, 1970. MR 41:333

11. S. Gao, Some applications of the Adams-Kechris technique, preprint, 2000.

12. J. Glimm, Locally compact transformation groups, Trans. Amer. Math. Soc., 101, 124-138, 1961. MR 25:146

13. V.V. Gorbatsevich, A.L. Onishchik, and E.B. Vinberg, Foundations of Lie Theory and Lie Transformation Groups, Springer, 1997. MR 99c:22009

14. L. Harrington, A.S. Kechris, and A. Louveau, A Glimm-Effros dichotomy for Borel equivalence relations, J. Amer. Math. Soc., 3 (4), 903-928, 1990. MR 91h:28023

15. G. Hjorth, Around nonclassifiability for countable torsion-free abelian groups, preprint, 1998.

16. G. Hjorth and A.S. Kechris, Borel equivalence relations and classifications of countable models, Ann. Pure and Appl. Logic, 82, 221-272, 1996. MR 99m:03073

17. J.E. Humphreys, Linear Algebraic Groups, Grad. Texts in Math., 21, Springer-Verlag, 1975. MR 53:633

18. S. Jackson, A.S. Kechris, and A. Louveau, Countable Borel equivalence relations, preprint, 2000.

19. A.S. Kechris, The structure of Borel equivalence relations in Polish spaces, Set Theory of the Continuum, H. Judah, W. Just, and W.H. Woodin (eds.), MSRI Publications, SpringerVerlag, 26, 89-102, 1992. MR 94h:03093

20. A.S. Kechris, Countable sections for locally compact group actions, Erg. Th. and Dyn. Syst., 12, 283-295, 1992. MR 94b:22003

21. A.S. Kechris, Classical Descriptive Set Theory, Grad. Texts in Math., 156, Springer-Verlag, 1995. MR 96e:03057

22. A.S. Kechris, Actions of Polish groups and classification problems, preprint, 1998.

23. A.S. Kechris, New directions in descriptive set theory, Bull. Symb. Logic, 5 (2), 161-174, 1999.

24. A.S. Kechris, Descriptive dynamics, Descriptive Set Theory and Dynamical Systems, Ed. by M. Foreman, A.S. Kechris, A. Louveau, and B. Weiss, London Math. Society Lecture Note Series, 277, 231-258, Cambridge Univ. Press, 2000.

25. A. Louveau and B. Velickovic, A note on Borel equivalence relations, Proc. Amer. Math. Soc., 120, 255-259, 1994. MR 94f:54076

26. G.A. Margulis, Discrete Subgroups of Semisimple Lie Groups, Erg. der Math. und Ihrer. Grenz., 17, Springer-Verlag, 1991. MR 92h:22021

27. C.C. Moore, Ergodic theory and von Neumann algebras, Proc. Symp. Pure Math., Amer. Math. Soc., 38, 179-226, 1982. MR 84f:22013

28. Y.N. Moschovakis, Descriptive Set Theory, North Holland, 1980. MR 82e:03002

29. J.J. Rotman, An Introduction to the Theory of Groups, Grad. Texts in Math., 148, SpringerVerlag, 1995. MR 95m:20001

30. K. Schmidt, Algebraic Ideas in Ergodic Theory, CBMS Regional Conference Series in Math., 76, Amer. Math Soc., 1990. MR 92k:28029

31. J.-P. Serre, A Course in Arithmetic, Grad. Texts. in Math., 7, Springer-Verlag, 1973. MR 49:8956

32. J. Silver, Counting the number of equivalence classes of Borel and co-analytic equivalence relations, Ann. Math. Logic, 18, 1-28, 1980. MR 81d:03051

33. S. Thomas, Notes on $\mathrm{TFA}_{2}$ groups, preprint, 1998. 
34. V.S. Varadarajan, Groups of automorphisms of Borel spaces, Trans. Amer. Math. Soc., 109, 191-220, 1963. MR 28:3139

35. R. Zimmer, Ergodic Theory and Semisimple Groups, Birkhäuser, 1984. MR 86j:22014

36. R. Zimmer, Groups generating transversals to semisimple Lie group actions, Israel J. Math., 73 (2), 151-159, 1991. MR 93e:22012

Department of Mathematics, University of Minnesota, Minneapolis, Minnesota 55455

E-mail address: adams@math.umn.edu

Department of Mathematics, Caltech, Pasadena, California 91125

E-mail address: kechris@caltech.edu 